\title{
Phenotype and multi-omics comparison of Staphylococcus and Streptococcus uncovers pathogenic traits and predicts zoonotic potential
}

Niels A. Zondervan', Vitor A. P. Martins dos Santos ${ }^{1,2}$, Maria Suarez-Diez ${ }^{1+}$ and Edoardo Saccenti ${ }^{1 *+}$ (D)

\begin{abstract}
Background: Staphylococcus and Streptococcus species can cause many different diseases, ranging from mild skin infections to life-threatening necrotizing fasciitis. Both genera consist of commensal species that colonize the skin and nose of humans and animals, and of which some can display a pathogenic phenotype.

Results: We compared 235 Staphylococcus and 315 Streptococcus genomes based on their protein domain content. We show the relationships between protein persistence and essentiality by integrating essentiality predictions from two metabolic models and essentiality measurements from six large-scale transposon mutagenesis experiments. We identified clusters of strains within species based on proteins associated to similar biological processes. We built Random Forest classifiers that predicted the zoonotic potential. Furthermore, we identified shared attributes between of Staphylococcus aureus and Streptococcus pyogenes that allow them to cause necrotizing fasciitis.

Conclusions: Differences observed in clustering of strains based on functional groups of proteins correlate with phenotypes such as host tropism, capability to infect multiple hosts and drug resistance. Our method provides a solid basis towards large-scale prediction of phenotypes based on genomic information.
\end{abstract}

Keywords: Staphylococcus, Streptococcus, Multi-omics, Comparison, Pathogenic, Traits, Prediction, Phenotype, Host-trophism, Zoonotic

\section{Background}

Species from the genera Staphylococcus and Streptococcus are mostly commensals that live as part of the microbiota of various animals and humans [1]. Some of them are opportunistic pathogens, displaying a pathogenic phenotype when the immune system of the host is compromised or the epithelial barrier is damaged [2-5].

\footnotetext{
* Correspondence: edoardo.saccenti@wur.nl

${ }^{+}$Maria Suarez-Diez and Edoardo Saccenti these authors jointly supervised this work.

${ }^{1}$ Laboratory of Systems and Synthetic Biology, Wageningen University \& Research, Stippeneng 4, 6708WE Wageningen, Netherlands

Full list of author information is available at the end of the article
}

Few comparative genomic studies have been performed to analyse the evolution and the pathogenesis of Staphylococcus and Streptococcus species: the comparisons of the genomes of 11 Staphylococcus species determined that horizontal gene transfer of virulence factors is an important factor in adaptation of $S$. aureus to humans [6]; another study showed that protein domain based metabolic diversity among Streptococcus species could be used to identify differences in the metabolism of the highly pathogenic serotype 2 S. suis compared to other Streptococci [7]. Another study confirmed these results and showed that metabolic capability predicted using genome scale models (GEMs)

(C) The Author(s). 2021, corrected publication 2021. Open Access This article is licensed under a Creative Commons Attribution 4.0 International License, which permits use, sharing, adaptation, distribution and reproduction in any medium or format, as long as you give appropriate credit to the original author(s) and the source, provide a link to the Creative Commons licence, and indicate if changes were made. The images or other third party material in this article are included in the article's Creative Commons licence, unless indicated otherwise in a credit line to the material. If material is not included in the article's Creative Commons licence and your intended use is not permitted by statutory regulation or exceeds the permitted use, you will need to obtain permission directly from the copyright holder. To view a copy of this licence, visit http://creativecommons.org/ licenses/by/4.0/. The Creative Commons Public Domain Dedication waiver (http://creativecommons.org/publicdomain/zero/1. 0/) applies to the data made available in this article, unless otherwise stated in a credit line to the data. 
could be used to identify Streptococcus strain specific biomarkers and metabolic determinants of virulence [8].

Protein domains and protein-domain architectures have been shown to be a fast and efficient method to define groups of functionally equivalent proteins that were used for comparative genomic studies $[9,10]$, including Staphylococcus and Streptococcus [11-13]. However, at the best of our knowledge, no work exists focusing on similarities and differences within and between Staphylococcus and Streptococcus genomes.

In this study we performed a comparative analysis of 235 and 315 fully sequenced Staphylococci and Streptococci genomes by annotating their proteins based on their domain content. We integrated this protein annotation with genome-scale metabolic-modelling predictions, transcriptomic and transposon-mutagenesis data sets to study gene essentiality and persistence. All annotation used in this paper as well as GO information is based on genomics annotation from databases based mainly on bacterial genomics studies. In this paper we compare within and between Staphylococcus and Streptococcus species with the objective to identify both difference and similarities in genomic properties as well as in specific combinations of genes that give rise to pathogenic phenotypes. We compared the clustering of Staphylococcus and Streptococcus genomes based on proteins selected using on Gene Ontology (GO) terms associated with clinical phenotypes such drug resistance, pathogenesis, and tissue and host tropism. Furthermore, we used the functional grouping of proteins to predict zoonotic potential of $S$. suis and S. agalactiae, that is their ability to infect multiple hosts including humans. Finally, we compared S. aureus and S. pyogenes to identify the genomic basis for their shared ability to cause severe bacterial infections like necrotizing fasciitis. Our results are compared throughout the paper with findings from literature.

\section{Results}

Pan- and core genome analysis

The size of the pan- and core genomes of Staphylococcus and Streptococcus was determined based on protein domain content (Fig. 1). The pangenome contains all proteins present in the analysed genomes. The core genome contains only proteins that are present in all genomes and represents their genomic essence [14]. The ratio of the sizes of the core- and pan genome are 0.22 (557/

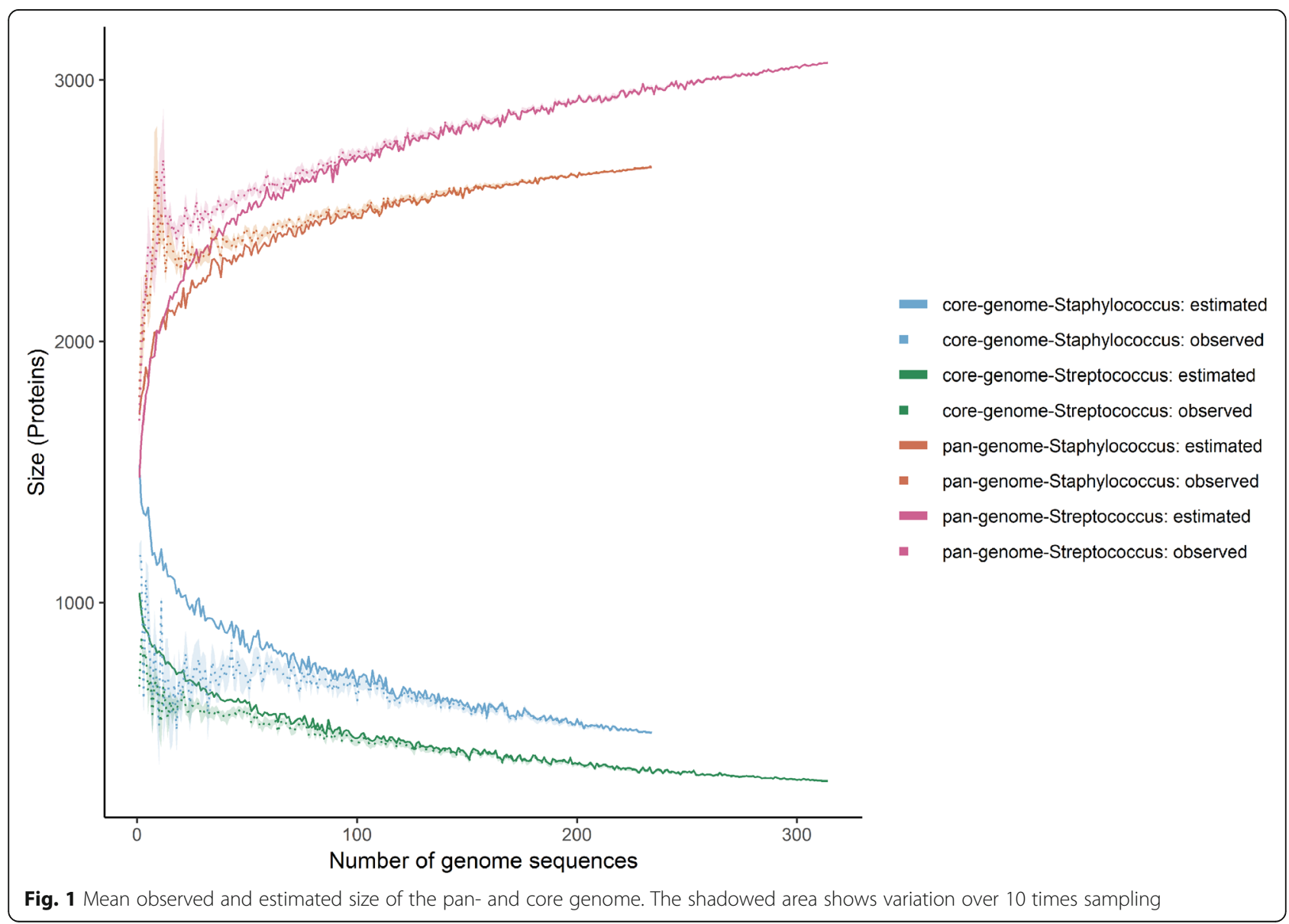


2563) for Staphylococcus and $0.17(458 / 2725)$ for Streptococcus.

A Heaps' regression model was used to estimate the closedness of the pangenome [15]. The closedness of the pangenome represents how much the addition of more genome sequences is expected to increase the number of proteins in the pangenome. For both Staphylococcus $(\alpha=1.10 \pm 0.02)$ and Streptococcus $(\alpha=1.12 \pm 0.01)$ the pangenome was found to be closed (i.e few new genes are added as news strains are discovered/sequenced). Additional plots of the estimated pan- and core genomes size and the Heaps' regression model can be found in supplementary material (see Additional file 5).

\section{Protein persistence and essentiality}

Persistence of proteins over all Staphylococcus and all Streptococcus species was calculated. Protein persistence data was combined with model predictions of essentiality and experimentally determined essentiality data.Experimentally determined essentiality (labelled as EXP) is available for growth on rich media resembling in vivo conditions. GEMs predictions were made using minimal media conditions for all combinations of carbon, nitrogen, sulphur, and phosphorus sources. Simulations on rich media conditions were therefore indirectly performed since all rich media compounds are present in the models as exchange reactions and all combinations of these exchange reactions functioning as carbon, nitrogen, sulphur and phosphor sources were tested for essentiality. We used GEM to predict gene essentiality for Staphylococcus aureus NTCTC 8325 and Streptococcus pyogenes M49. The total number of medium combinations based on $C$, N, S, P sources was 12,432 for Staphylococcus and 714 for Streptococcus. The number of tested conditions for Staphylococcus is much larger than the Streptococcus model since the Staphylococcus model can use all amino acids as alternative nitrogen source through deamination, greatly increasing the number of minimal media combinations. The Staphylococcus model can use all amino acids as alternative nitrogen source through deamination, greatly increasing the number of minimal media combinations Protein persistence, in silico predictions of essential and in vitro essentiality data for Staphylococcus and Streptococcus were integrated based on their associated locus tags. Both GEM based and experimentally determined essentiality correlated with a high persistence, while essentiality by both criteria is associated with an even higher persistence (see Table 1 and Fig. 2). Proteins experimentally determined or GEM predicted to be essentiality are significantly different from the average protein persistence (Student's $t$-test, $p$-value $=5 \times$ $10^{-14}$ ) for both Staphylococcus and Streptococcus.
Table 1 Persistence of Staphylococcus (Staph.) and Streptococcus (Strep.) for all proteins, proteins associated to Genome Metabolic model (GEM) essential genes and experimentally (EXP) determined essential genes

\begin{tabular}{lll}
\hline Group & Avg persistence Staph. & Avg persistence Strep. \\
\hline All & $0.60 \pm 0.44(N=2655)$ & $0.42 \pm 0.42(N=3047)$ \\
GEM-essential & $0.94 \pm 0.14(N=153)$ & $0.98 \pm 0.09(N=225)$ \\
Exp-Essential & $0.97 \pm 0.03(N=411)$ & $0.97 \pm 0.12(N=254)$ \\
EXP\&GEM-Essential & $0.94 \pm 0.01(N=46)$ & $0.98 \pm 0.00(N=113)$ \\
\hline
\end{tabular}

Variability of gene expression and gene essentiality

Essentiality and domain persistence information for Staphylococcus was combined with the variability of transcription (measured by $\log _{2}$ fold changes). The variability in expression for experimentally determined essential and non-essential genes as well as for persistent and non-persistent genes were compared (Fig. 3). The fold change transcription levels of experimentally determined essential genes are significantly less variable than the transcription levels of non-essential genes (Student's $t$-test, $p$-value $=5 \times 10^{-14}$ ) as well as for persistent genes (Student's $t$-test, $p$-value $=0.000124)$ ).

\section{Functional analysis of pathogenesis and pathogenicity}

For this analysis, we filtered proteins from Staphylococcus and Streptococcus on their association to 17 genome ontology (GO) biological process terms associated to pathogenesis and pathogenicity but cannot make predictions for rich media conditions were not all components are known or incorporated in the model. Filtering included all proteins associated to either the 17 main GO terms or any of their descendent terms. For all GO terms, proteins were found in both Staphylococcus and Streptococcus (Table 2). The ratio of proteins per GO function to the total number of proteins is similar for Staphylococcus and Streptococcus except for the group 'Biological adhesions' which has a larger fraction of proteins associated in Streptococcus than in Staphylococcus.

Functional trees, PCA and t-SNE plots were used to compare the (dis-)similarity in clustering of the genomes based on functional groups of proteins compared to clustering based on all proteins. Dissimilarity was calculated using the Euclidean distances of genomes in the functional trees and by scaling these distances to values between 0 and 1 to make them comparable.

Functional trees, PCA plots and t-SNE plots for Staphylococcus can be found in supplementary material [Additional files 6, 7 and 8]. Functional trees, PCA plots and t-SNE pots for Streptococcus can be found in supplementary material (Additional files 9, 10 and 11). PCA plots and t-SNE plots for Staphylococcus and Streptococcus species combined can be found in supplementary material (Additional files 12 and 13). 
A

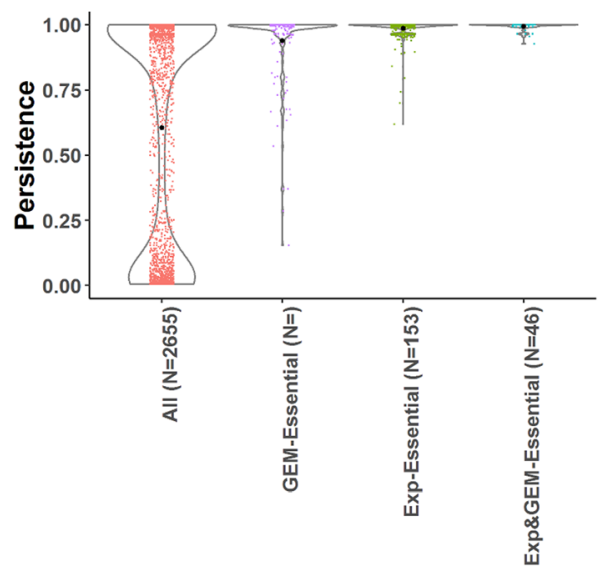

B

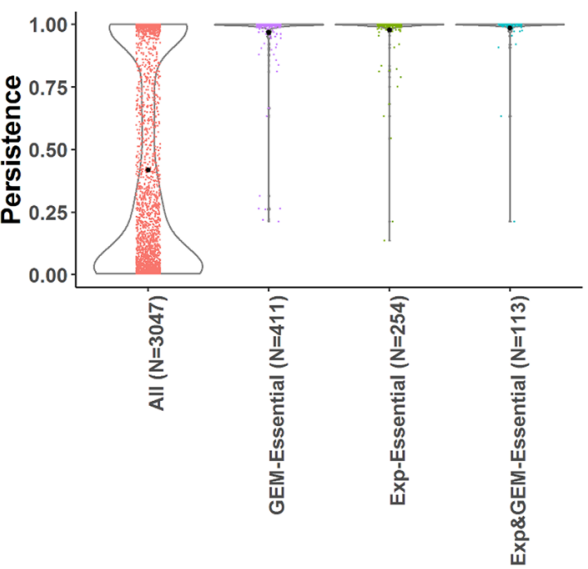

Fig. 2 Protein persistence. a Staphylococcus, b Streptococcus. Group labels: All = all proteins, GEM=in silico predicted to be essential using a Genome Scale Metabolic model, Exp-Essential = experimentally determined to be essential. Combined group strains

Heatmaps were used to investigate which proteins are absent for each species. Each of these analyses and visualization methods has their own strength and weaknesses in showing the differences in clustering. In the following we highlight some of the differences in clustering of Staphylococcus and Streptococcus genomes based on proteins annotated per GO term as compared to clustering based on all proteins.

\section{Correlation between GO functional groups of proteins}

We calculated the correlation between functional trees in order to compare the similarity in clustering per GO functional group of proteins (Fig. 4a-b). The correlation between functional trees is higher for children and parent
GO terms as well as for GO terms with similar functions such as 'drug metabolic process 'and 'response to drug'. In general, we see that functional trees based on fewer proteins have a lower correlation than functional trees based on many proteins. These results were expected since fewer proteins means less information to separate strains resulting in merging of branches in the tree. An interesting exception to this rule is the 'symbiont process' functional tree which has the lowest correlation with other functional trees for Staphylococcus even though there is a high number of proteins associated to this GO term.

There are some notable differences when comparing the correlation between functional trees for Staphylococcus and Streptococcus. For Staphylococcus, the 'pathogenesis'
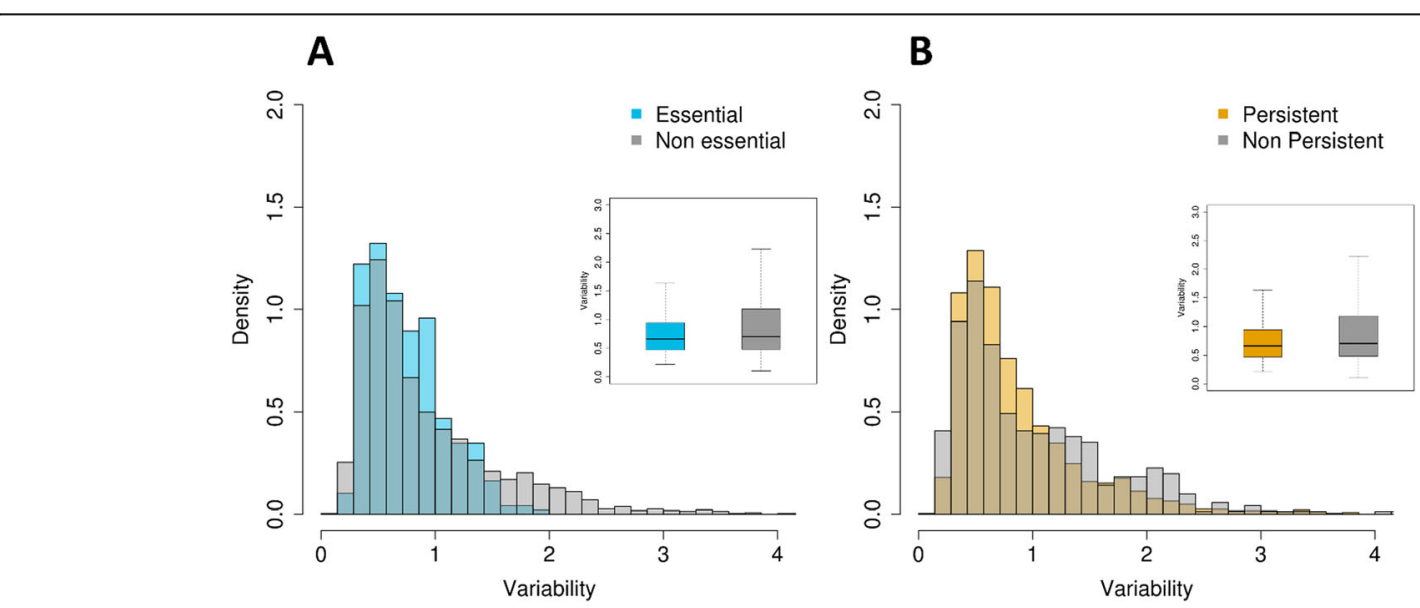

Fig. 3 a Transcriptional variability of essential and non-essential genes in Staphylococcus. Box plots show Variability values for both groups. Difference between mean values is significant $(p$-val $<0.01)$. $\mathbf{b}$ Transcriptional variability of persistent and non-persistent genes (genes with persistence lower or higher than 0.95 , respectively). Box plots show Variability values for both groups. Difference between mean values is significant $(p$-val $<0.01)$ 
Table 2 Number of proteins in the pangenome of Staphylococcus and the pangenome of Streptococcus per GO term. Root ontology terms, terms without a parent, are marked in their description with an asterixis $\left(^{*}\right)$. GO terms are order as such that descendent GO terms are shown below their parent

\begin{tabular}{|c|c|c|c|}
\hline Filter & Description & Staph & Strep \\
\hline & All proteins & 2655 & 3047 \\
\hline GO:0008150 & Biological process & 1974 & 2222 \\
\hline GO:0008152 & ${ }^{*}$ Metabolic process & 1661 & 1871 \\
\hline GO:0017144 & Drug metabolic process & 59 & 77 \\
\hline GO:0042493 & Response to drug & 56 & 60 \\
\hline GO:0023052 & *Signalling & 217 & 280 \\
\hline GO:0065007 & *Biological regulation & 823 & 929 \\
\hline GO:0022610 & *Biological adhesion & 70 & 147 \\
\hline GO:0044406 & Adhesion of symbiont to host & 2 & 3 \\
\hline GO:0051704 & Multi-organism process & 348 & 456 \\
\hline GO:0044419 & Inter species interaction between organisms & 210 & 309 \\
\hline GO:0042710 & Biofilm formation & 9 & 10 \\
\hline GO:0098743 & Cell aggregation & 7 & 10 \\
\hline GO:0044403 & Symbiont process & 150 & 202 \\
\hline GO:0009372 & Quorum sensing & 7 & 7 \\
\hline GO:0035821 & Modification of morphology or physiology of other organism & 45 & 74 \\
\hline GO:0009405 & Pathogenesis & 65 & 115 \\
\hline
\end{tabular}

clusters together with the functional tree 'modification of morphology or physiology of other organisms'. For Streptococcus, the functional tree of 'pathogenesis' clusters together with the functional tree of 'biological adhesion'. Many 'modification of host morphology' proteins in Staphylococcus are also associated to the GO term 'pathogenesis' while many 'biological adhesion' proteins in Streptococcus are associated to the GO term 'pathogenesis'. These results could indicate that modification of host morphology is important for the pathology of Staphylococcus strains while biological adhesion is more important for the pathology of Streptococcus.

\section{Horizontal gene transfer of proteins related to pathogenesis}

The PCA plot based on all proteins combining Staphylococcus and Streptococcus genomes supplementary material (Additional files 12 and 13), shows genomes of the same species to cluster together as we would expect (Fig. 5a). The PCA plot based on presence/absence of proteins involved in Response to drug (GO:0042493) shows genomes are not always separated on the species level, however, there is a clear separation between Staphylococcus and Streptococcus genomes (Fig. 5b). However, both in the PCA (Fig. 5c) and in t-SNE plots based on proteins associated to the GO term 'Pathogenesis' proteins, Staphylococcus and Streptococcus species cluster together.
Analysis of the presence and absence of proteins associated to 'pathogenesis' reveals that Staphylococcus sciuri GCA:002072755 and Staphylococcus haemolytic us GCA: 001611955 only contain one pathogenesis protein (PF04647) that is not present in any Streptococcus strain. This protein, PF04647 ArgB, is part of a quorum sensing system. Also S. saprophyticus GCA:002209265 only contains one protein not present in any Streptococcus strain. This protein, PF05480, is a haemolytic protein unique to Staphylococcus. Among Streptococci, S. parauberis six $S$. iniae and seven $S$. thermophilus strains lack any pathogenesis protein that separates them from Staphylococcus.

\section{Domain shuffling of pathogenic proteins}

The Staphylococcus pangenome contains 52 domains present in 65 proteins associated to the GO term 'pathogenesis' while the Streptococcus pangenome contains 88 domains present in 118 proteins associated to the GO term 'pathogenesis'. 20\% of pathogenic proteins in Staphylococcus and 25\% of the pathogenic proteins in Streptococcus consist of a few pathogenesis associated domains combined with domains not directly associated to pathogenesis. This implies that domain shuffling might be an important evolutionary factor for these pathogens. In Staphylococcus 46\% (30/65) and in Streptococcus $72 \%(85 / 118)$ of the pathogenesis associated proteins contain multiple domains. This percentage is much higher than the average percentage of multidomain proteins of 8.9 and $9.2 \%$ for Staphylococcus and 

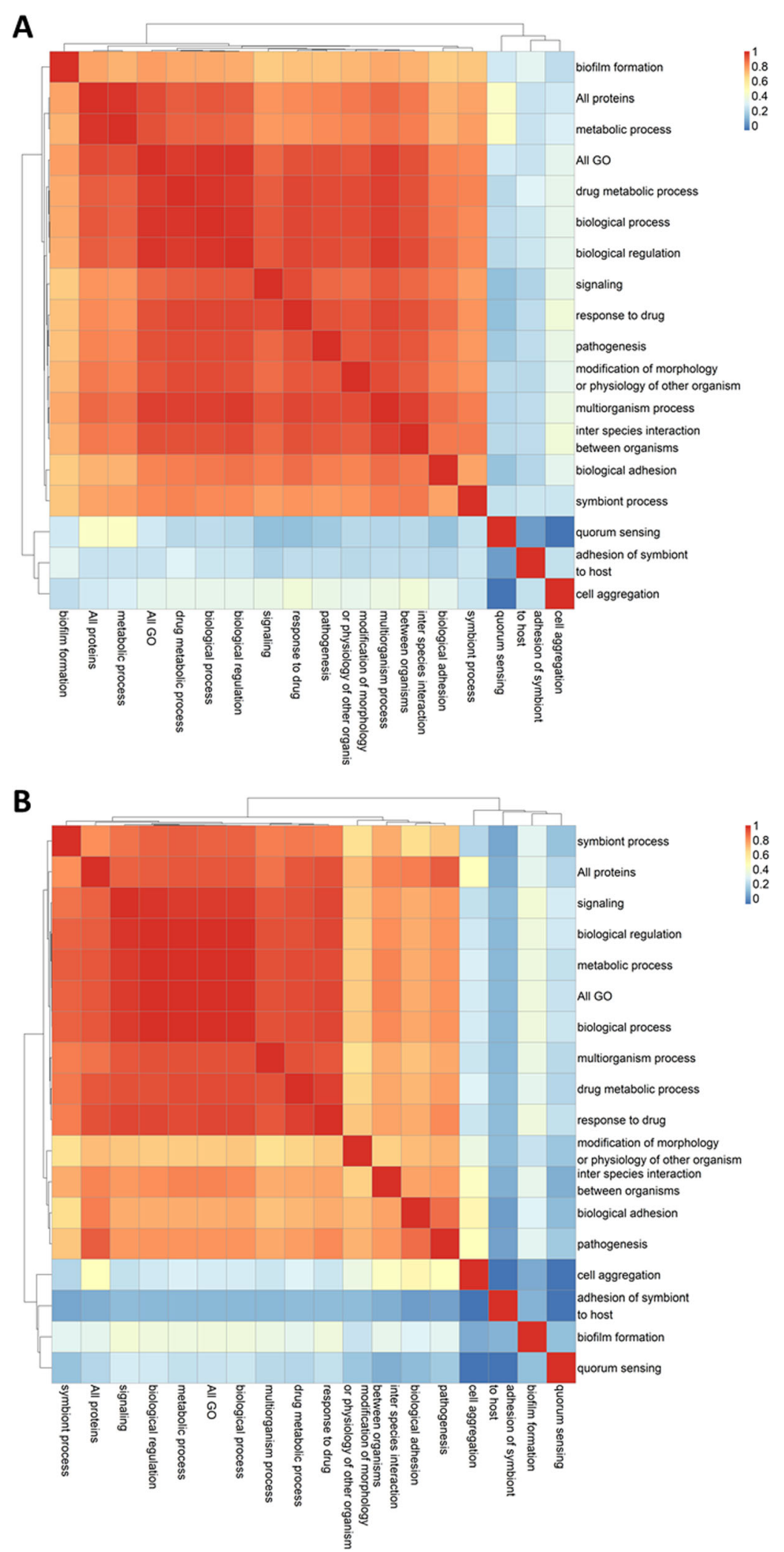

Fig. 4 a Heatmaps of the correlation between Staphylococcus functional trees, $\mathbf{b}$ Heatmaps of the correlation between Streptococcus functional trees 


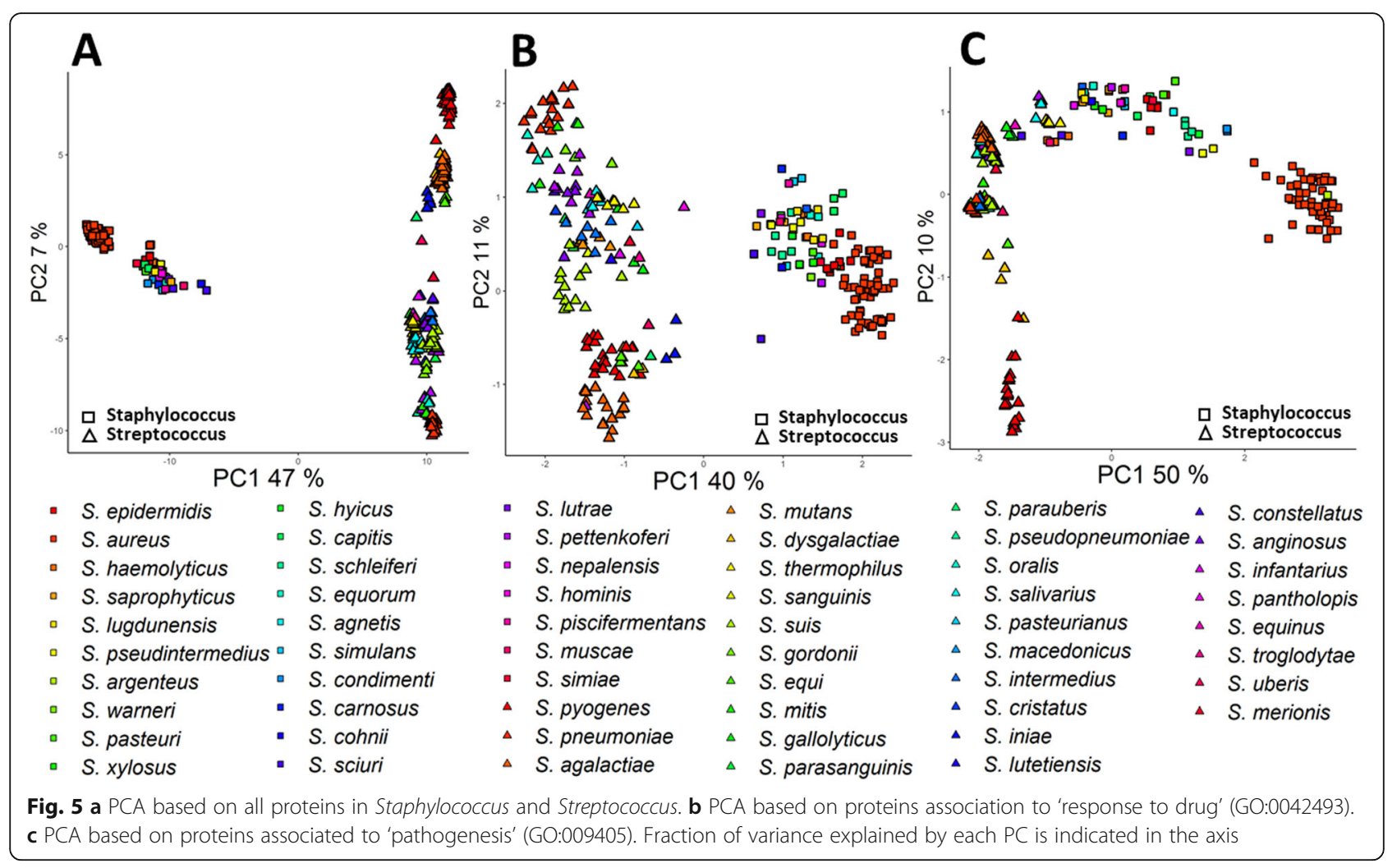

Streptococcus respectively. It could be argued that proteins involved in pathogenesis would more often require multiple domains since many of them are cell-wall associated, secreted or contain multiple domains to facilitate interaction between host and the pathogen. The importance of cell wall associated proteins is reflected by the high percentage of $40 \%$ of pathogenesis proteins in Staphylococcus and 66\% Streptococcus that contain LPXTG cell-wall anchor domain PF00746. The importance of this domain for pathogenesis was shown in a $S$. aureus mutant with a knockout of $s r t A$ coding for a class A sortase, which is required for secretion of proteins containing the LPXTG motif. This mutant was unable to form abscess lesions in organ tissues or cause lethal bacteraemia when inoculated in the blood stream of mice [16].

\section{Staphylococcus aureus multi-drug resistance}

We investigated the clustering of S. aureus genomes in the functional tree associated to the terms "response to drug". We selected the genome of $S$. aureus sub species aureus MRSA 252 (GCA:000011505), which is known to be a multiple drug resistant strain [17]. Next, we searched literature for information about drug resistance for eight genomes that cluster together with this strain in the functional tree response to drug. For seven of these strains (JH1, JH9, Mu50, Mu3, T0131, 04-20,981), evidence was found for these strains to be multi-drug resistant as well as identifying two pathogenicity islands as the cause of their resistance [18-22]. For the last genome (GCA:001640885), no literature or other information could be retrieved. This genome has exactly the same proteins associated to response to drug as the seven strains for which multi drug resistance was reported in literature. Therefore, we can speculate that this strain may have the same multi drug resistance phenotype.

\section{Streptococcus suis pathogenesis zoonotic potential}

Large differences in clustering were observed for S. suis genomes in the functional trees relating to 'biological adhesion', 'modification of morphology or physiology of other organism' and 'pathogenesis', supplementary material (Additional file 9). S. suis genomes form two groups in the functional tree of biological adhesion, and three groups in the functional tree of pathogenesis and 'modification of morphology or physiology of other organism'.

Similarly, different groups can be distinguished in the PCA plot based on these three functional groups, as shown in Fig. 6a-c. We included information from literature on zoonotic species, namely $S$. inae, S. agalactiae, $S$. dysgalactiae S. iniae and, S. equi zooepidemicus and $S$. suis serotype 2 strains and serotype information and host isolation information for $S$. suis and S. agalactiae strains in the labels of Fig. 6a-c. Two S. suis clusters can be distinguished in the PCA score plot based on proteins 


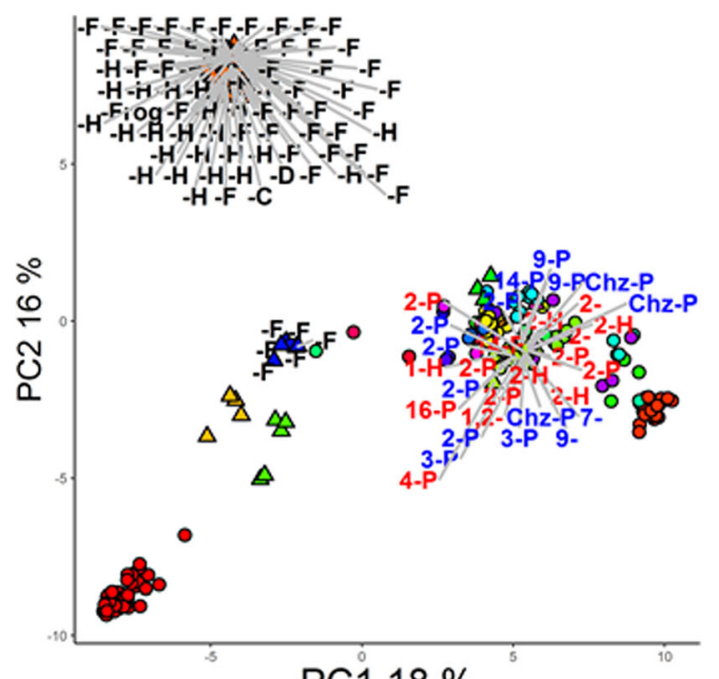

A S.pyogenes

S. pneumoniae

S. agalactiae

S. mutans

S. dysgalactiae

S. thermophilus

S. sanguinis

O S. suis

S. gordonii

S. equi

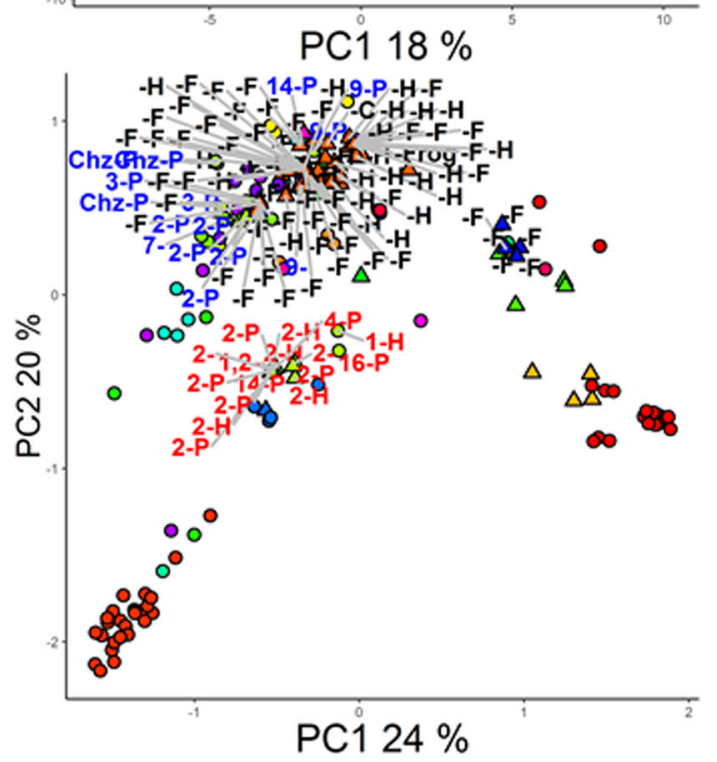

S. mitis

S. gallolyticus

S. parasanguinis

B

S. parauberis

S. pseudopneumoniae

S. oralis

S. salivarius

S. pasteurianus

S. macedonicus

S. intermedius

- S. cristatus

- S. iniae

- S. lutetiensis

- S. constellatus

- S. anginosus

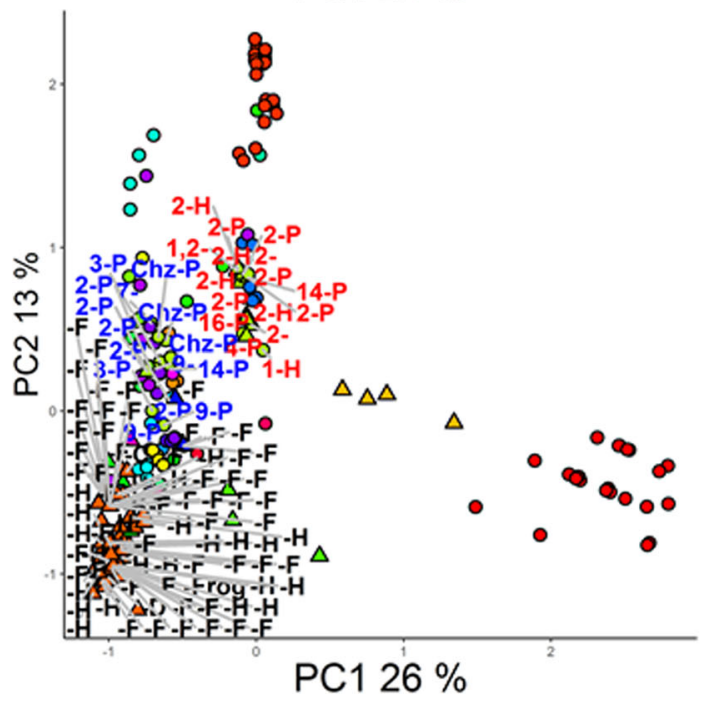

S. infantarius

C

S. pantholopis

S. equinus

S. troglodytae

S. uberis

S. merionis

\section{O non-zoonotic \\ $\Delta$ zoonotic}


(See figure on previous page.)

Fig. 6 PCA plot of Streptococcus strains based on all proteins (a), proteins filtered on 'modification of morphology or physiology of other organism' (b) and proteins filtered on 'pathogenesis' (c). S. suis serotypes are shown in the label, genomes from species mentioned in literature as having zoonotic capabilities are marked with a triangle and the isolation host is marked in the label with $\mathrm{D}=\mathrm{dog}, \mathrm{F}=$ fish, $\mathrm{H}=\mathrm{human}, \mathrm{P}=$ pig,

$\mathrm{T}=$ toad. Genomes predicted in this study to have zoonotic potential are coloured red while strains in the cluster predicted not to have zoonotic potential are coloured blue. Fraction of variance explained by each PC is indicated in the axis

related to 'modification of morphology or physiology of other organism' (Fig. 6b) and the PCA based on 'pathogenesis' proteins (Fig. 6c): the first cluster contains 7 out of the 12 serotype 2 strains, as well as serotype $1,1,2,4$, 16 , while the second cluster contains 5 serotype 2 strains as well as strains with serotype 3, 7, 914 and Chz which were all isolated from pigs. The first group contains $S$. suis zoonotic strains of which some are isolated from pig and some from humans. The second group contains are non-zoonotic strains all isolated from pigs.

\section{Streptococcus agalactiae zoonotic potential}

Like $S$. suis, S. agalactiae forms two clusters when clustering on $\mathrm{GO}$ biological functional groups of proteins. Based on their isolation host, we can see that a cluster contains strains that are zoonotic while the other contains strains that are non-zoonotic. These two groups of $S$. agalactiae strains are better separated when using t- $t$ SNE plots based on all proteins and proteins involved in biological adhesion and pathogenesis (Fig. 7a-c) suggesting the existence of few proteins that are present in every genome in each group.

\section{Identification of proteins that confer zoonotic potential}

We used Random Forest, a machine learning approach, to investigate the association between genome content and phenotype using $75 \%$ of the data for training and $25 \%$ of the data for validation. Specifically, presence/absence of proteins filtered on association to GO Biological functions involved in pathogenesis to predict zoonotic potential $S$. suis and $S$. agalactiae, and we investigated which proteins are responsible for the zoonotic potential in these two species. We used functional groups of proteins that were shown to separate zoonotic and nonzoonotic strains for S. suis (Fig. 6b-c) and for S. agalactiae (Fig. 7b-c) to train a Random Forest classifiers. We investigated their overall importance for prediction as well as their contribution to predicting the class nonzoonotic, and the class zoonotic potential as shown in Fig. 8a-d. Where, the 'Impact' measure indicates the relevance of a protein of the prediction of given class. The 'importance' shows the proteins overall importance for the random forest classifier. Random Forest classifiers as well as the optimal hyper parameters can be found in (see Additional file 14).

The protein domain content of the five most important proteins for $S$. suis classification based on 'modification of morphology or physiology of other organism' proteins are: 1) PF01289 a thiol-activated cytolysin, 2) PF17440 thiol-activated cytolysin beta sandwich domain, 3) PF00910 replication initiation protein involved in viral RNA duplication 3) PF00078;PF08388; PF13655 group II intron reverse transcriptase/maturase, 4) PF03432 a relaxase involved in transfer of plasmids, 5) PF00665 Prokaryotic N-terminal methylation motif often found in pilins and other proteins involved in secretion (Fig. 8a).The most important proteins for S. suis classification based on 'pathogenesis' proteins are 1) PF01289 thiol-activated cytolysin, 2) PF17440 a thiolactivated cytolysin beta sandwich domain, 3) PF07564 hypothetical protein containing a domain of unknown function, 4) PF00092; PF00746 chemotaxin protein 5) PF00746;PF08363;PF16364 a glucan binding protein (Fig. 8b).

The S. suis classifiers based on 'modification of morphology or physiology of other organism' proteins as well the classifier based on 'pathogenesis' proteins, predict $S$. suis zoonotic potential with $100 \%$ accuracy solely based on the presence of either PF01289, a thiol-activated cytolysin or PF17440, a thiol-activated cytolysin beta sandwich Fig. 8a-b).

The most important features for $S$. agalactiae classification based on 'biological adhesion' proteins are 1) PF02872 a 5'-nucleotidase-C 2) PF00746;PF08017 Fibrinogen binding protein A, 3) PF00746; PF8428; PF08829; PF174802 surface protein Rib and 4) PF00746; PF16555; PF16569; PF16570 pilus complex 5) PF00746; PF11966 a cell wall anchored linked to a ySIRK signal domain (Fig. 8c). The most important features for $S$. agalactiae classification based on 'pathogenesis' proteins are 1) PF00746;PF08017 Fibrinogen binding protein, 2) PF00746;PF8428;PF08829;PF17480 2) surface protein Rib and 3) PF00746;PF16555;PF16569;PF16570 pilus complex 4) PF00746;PF11966 a cell wall anchored linked to a ySIRK signal domain 5) PF02225;PF07554, a serine protease (Fig. 8d).

For $S$. agalactiae classification, $5^{\prime}$-nucleotidase-C can predict training data with $100 \%$ accuracy and test data with $94 \%$ accuracy.

\section{Virulence factors of necrotising fasciitis}

We compared Staphylococcus aureus and Streptococcus pyogenes since both are a major cause of (monomicrobial) necrotizing fasciitis. Both $S$. aureus [23-25] and $S$. 


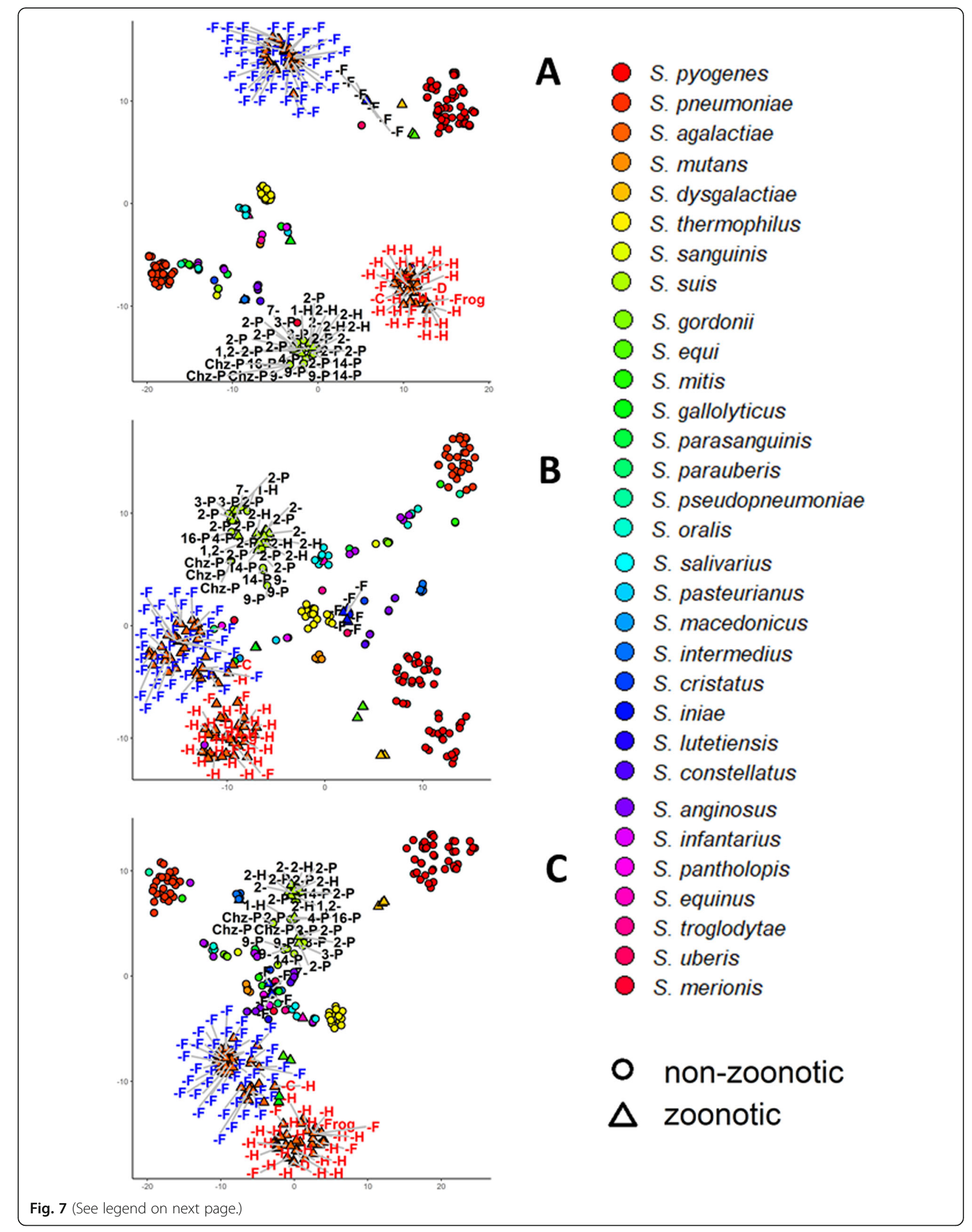


(See figure on previous page.)

Fig. 7 t-SNE plots of Streptococcus strains based on proteins all proteins (a), proteins filtered on 'biological adhesion' (b) and 'pathogenesis' (c). tSNE is a technique for dimensional reduction and visualization, so that similar objects appear as nearby objects in the two-dimensional plots here presented. S. suis serotypes are shown in the label, genomes from species mentioned in literature as having zoonotic capabilities are marked with a triangle and the isolation host is marked in the label with $\mathrm{D}=\mathrm{dog}, \mathrm{F}=$ fish, $\mathrm{H}=$ human, $\mathrm{P}=$ pig, $\mathrm{T}=$ toad. Genomes predicted in this study to be part of the zoonotic potential cluster are coloured red while strains in the cluster predicted not to have zoonotic potential are coloured blue.

Fraction of variance explained by each PC is indicated in the axis

pyogenes $[26,27]$ fully lyse red blood cells, induce toxic shock syndrome as well as bind and invade epithelial host cells. Based on their GO term association $S$. aureus has 21 proteins associated to pathogenesis that occur in nearly all $S$. aureus genomes and only rarely in any other Staphylococcus specie (Table 3). Some of these pathogenesis proteins can directly be linked to pathogenesis proteins reported in literature for S. aureus [31] and for S. pyogenes [32]. An exact match with proteins reported in literature is however not always possible due to differences in annotation. When considering virulence factors that are not unique to $S$. aureus or $S$. pyogenes the number of virulence factors is about 1.5 times as many as reported in literature [33].

We looked at shared proteins as well as functional alternatives to find the molecular basis for necrotising fasciitis and we used the PFAM description of protein domains as well as description of proteins based on the locus tags associated to these proteins. We found that both $S$. aureus and S. pyogenes contain proteins involved in fibronectin binding, wound invasion, haemolysis, cell adhesion, IgA and IgG binding, multiple (super-)toxins as well as proteins involved in resisting phagocytosis and invading host cells (Table 3, Fig. 5). For example,
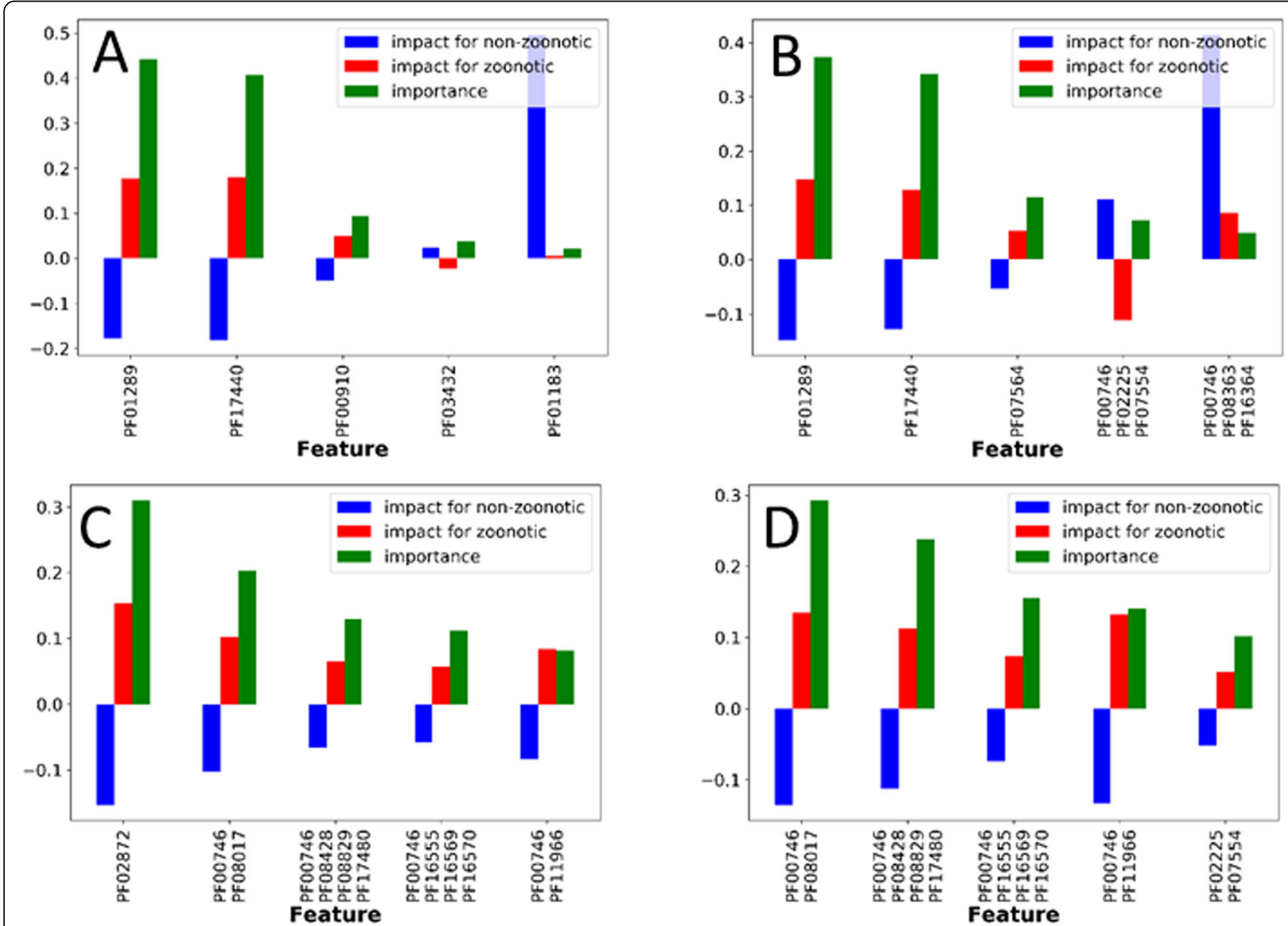

Fig. 8 Protein feature contribution to predict the class 'non-zoonotic' and 'zoonotic' as well as the overall importance of the protein feature for classification. a The five most important 'modification of morphology or physiology of other organism' proteins used to classify S. suis. $\mathbf{b}$ The five most important 'pathogenesis' proteins used to classify S. suis. c The five most important 'biological adhesion' proteins used to classify S. agalactiae. $\mathbf{d}$ The five most important 'pathogenesis' proteins used to classify S. agalactiae 
Table 3 Proteins associated to S. aureus Pathogenesis (GO: GO:0009405). Domains that are shared between S. aureus and S. pyogenes are underlined. Proteins that are shared have are written in bold

\begin{tabular}{|c|c|}
\hline Protein & Description \\
\hline 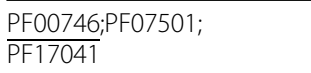 & LPXTG cell wall anchor; G5 domain, suggested adhesion, in peptide that cleaves IgA; E domain, rod like structure \\
\hline PF00746; PF17210 & LPXTG cell wall anchor, SdrD B-like domain, involved in adhesion to nose squamous cells [28] \\
\hline PF13545 & Crp-like helix-turn-helix domain, possibly cAMP interaction \\
\hline PF05543 & Staphopain peptidase $C 47$, secreted cysteine protease \\
\hline PF14731 & Staphopain proregion \\
\hline PF03373 & Octapeptide repeat, part of SpA virulence factor frequently used to type S. aureus strains [29] \\
\hline PF07968 & Haemolysin, part of the Leukocidin/Hemolysin toxin family \\
\hline PF09199 & $\begin{array}{l}\text { Staphylococcal superantigen-like OB-fold domain, interact with IgA, inhibits the end stage of complement activation } \\
\text { and IgA binding to Fc-a-R [30] }\end{array}$ \\
\hline PF02216 & SpAB protein domain, immunoglobin binding domain \\
\hline PF02876 & Staphylococcal/Streptococcal toxin, beta-grasp domain \\
\hline PF11621 & C3 binding domain 4 of lgG-bind protein SB \\
\hline PF01123 & $\begin{array}{l}\text { Enterotoxin type B, supertoxin, involved in food poisoning, causing the immune system to release a large number of } \\
\text { cytokines that lead to significant inflammation }\end{array}$ \\
\hline PF03642 & MAP domain, major histocompatibility complex class II analog \\
\hline PF00746;PF01476 & LPXTG cell wall anchor motif; LysM domain found in many receptors, peptidoglycan-binding protein [12] \\
\hline PF00746;PF02986 & LPXTG cell wall anchor motif; Fibronectin binding repeat, enables uptake by host cell \\
\hline PF00746;PF05031 & LPXTG cell wall anchor motif; Iron Transport-associated domain, heme and/or hemoprotein-binding \\
\hline PF07564 & $\begin{array}{l}\text { Domain of Unknown Function (DUF1542), several proteins containing this domain are involved in antibiotic resistance } \\
\text { and/or cell adhesion }\end{array}$ \\
\hline PF09023 & Staphostatin B inhibits the cysteine protease Staphopain B \\
\hline PF02821 & Streptokinase (SK) is a thrombolytic medication and enzyme, breaks down blood cloths \\
\hline PF01468 & $\begin{array}{l}\text { GA module, GA modules may promote bacterial growth and virulence in mammalian hosts by scavenging } \\
\text { albumin-bound nutrients and camouflaging the bacteria }\end{array}$ \\
\hline PF07554;PF07564;PF08428 & $\begin{array}{l}\text { FIVAR domain, likely binds fibronectin or more specifically N-acetyl glucosamine, occurs in proteins involved in methicillin } \\
\text { resistance; Domain of Unknown Function (DUF1542); Rib/alpha-like repeat. Occurs in some Rib, a thought to confer } \\
\text { protective immunity. Occurs in some Streptococcus surface proteins. Extracellular matrix-binding protein Ebh }\end{array}$ \\
\hline
\end{tabular}

PF01123 and PF02876 toxin $\beta$-grasp domain together form Enterotoxin type 2 which is important for causing the toxic shock $[4,34]$. Enterotoxin type 2 antibodies are currently in clinical trials tested and have shown potential in treating necrotizing fasciitis [35].

S. pyogenes has 10 proteins that present in all S. pyogenes species and only occur separately in a few other Streptococcus strains (Table 4, Fig. 5). Five of these proteins are associated to the ability of $S$. pyogenes to bind to and break down fibrin in blood cloths [27]. Other proteins include toxin and enterotoxin, involved in over-activation of the immune response [38, 39], fibrin binding proteins, involved in adhesion and intracellular access of host cells, as well as proteases, involved in resistance to phagocytosis [40]. S. pyogenes has fewer proteins that are unique to this species compared to S. aureus since many other Streptococcus strains produce some of the pathogenic proteins present in S. pyogenes [41].

Only three proteins associated to pathogenesis are shared between S. aureus and S. pyogenes, Enterotoxin B
C-terminal domain (PF02876), Enterotoxin B N-terminal beta-grasp domain (PF01123) and Fibronectin binding protein (PF00746;PF02986). Both fibronectin binding protein $\mathrm{A}(\mathrm{FnbpA})$ and $\mathrm{B}(\mathrm{FnbpB})$ are expressed during infection conditions and were shown to be complexly regulated by a large number of regulators such as sigma factors and two component systems by Mader et al. [42]. Among these proteins identified in our study are potential biomarkers. FnbpA was found not to be essential in KO studies [43], but was found to be essential in a rapid shotgun antisense RNA method to identify essential genes in S. aureus [44].. No essentiality information is available for FnbpB. S. aureus fibronectin binding protein $\mathrm{A}(\mathrm{FnbpA})$ is called fibronectin binding protein $\mathrm{X}$ (SfbX) in S. pyogenes. For S. aureus, FnbpA was found to be essential for entry in the host cells [45]. FnbpA has functional homologs in other species such as S. epidermidis, however all homologs lack the C-terminal multiple fibronectin binding repeats variants present in FnbpA, of which at least one high affinity binding repeat 
Table 4 Proteins associated to S. pyogenes Pathogenesis (GO: GO:0009405). Domains that are shared between S. aureus and S. pyogenes are underlined. Proteins that are shared are written in bold

\begin{tabular}{|c|c|}
\hline Protein & Description \\
\hline PF02821 & Streptokinase, breaks down blood cloths \\
\hline PF01640 & Peptidase C10 family \\
\hline$\underline{\text { PF01123 }}$ & Enterotoxin type $B$, super antigen involved in food poisoning \\
\hline$\underline{\text { PF02876 }}$ & Staphylococcal/Streptococcal toxin, beta-grasp domain \\
\hline PF03734 & L,D-transpeptidase catalytic domain, peptidoglycan binding \\
\hline$\underline{\text { PF00746; PF01391 }}$ & $\begin{array}{l}\text { LPXTG cell wall anchor motif; Collagen helix, rod like structure, coagulation-fibrinolytic binding in blood, } \\
\text { Scl1 adhesin specifically recognizes the wound microenvironment [36] }\end{array}$ \\
\hline$\underline{\text { PF00746; PF02370 }}$ & $\begin{array}{l}\text { LPXTG cell wall anchor motif; M protein repeat, binds IgA, major virulence factor involved in host cell } \\
\text { invasion and resistance to phagocytosis [37] }\end{array}$ \\
\hline$\underline{\text { PF00746; PF08017 }}$ & $\begin{array}{l}\text { LPXTG cell wall anchor motif; Fibronogen binding protein, members of this family include the fibrinogen } \\
\text { receptor, FbsA which mediates platelet aggregation }\end{array}$ \\
\hline PF00746; PF02986 & $\begin{array}{l}\text { LPXTG cell wall anchor motif; Fibronectin binding repeat, mediate adherence to host cells, enable the } \\
\text { colonisation of wound tissue and blood clots }\end{array}$ \\
\hline PF00092; PF00746; PF02986 & $\begin{array}{l}\text { Von Willebrand factor type A domain domains participate in numerous biological events (e.g. cell adhesion, } \\
\text { migration, homing, pattern formation, and signal transduction); LPXTG cell wall anchor motif; Fibronectin } \\
\text { binding repeat }\end{array}$ \\
\hline
\end{tabular}

is needed for host cell uptake [16, 34, 46]. A SfbX knockout mutants was shown to be only minimally affect $S$. pyogenes ability to infect epithelial host cells [47]. Enterotoxin type B, is a super toxin involved in over-activation of the immune response and interferes with phagocytosis by supressing the generation of myeloid-derived suppressor cells [48-50].

\section{Discussion}

The Staphylococcus and Streptococcus genera were compared on their genomic properties. Both genera have a similar ratio of their pan and core genome size. It should be considered that this analysis has been done with all fully assembled genomes data that were available at the time of the study. In our study we do not separate between pathogenic and non-pathogenic species since there are several ways to infect humans and animals. Instead, we use the underlying annotation of proteins marked as being involved in GO functions associated to pathogenesis to investigate patterns in pathogenesis. The choice not to define before-hand if species are pathogenic is deliberate since we recognize there are many forms of pathogenesis which depend on both species as well as the infection site as we discuss in the sections "Streptococcus suis pathogenesis zoonotic potential" and in the section "Streptococcus agalactiae zoonotic potential". We do however recognize the selected population affect our results as can be seen for in the ratio of their pan and core genome size found in this study. The alpha value of 1.12 found in our study for Streptococcus is higher than the 0.87 values reported by Koehorst et al. [12]. This difference can be explained by the number of genomes analysed which was 314 in our study opposed to 60 in the study by Koehorst et al. Additionally, we allowed a maximum of one genome per species to be selected in our sampling approach to avoid population bias introduced by species with many sequenced genomes such as $S$. aureus which was not the case in the analysis of by Koehorst et al. [12].

Similar to what was found for Pseudomonas [9], gene expression variability of essential genes was found to be less than the expression variability of non-essential genes in both $S$. aureus and $S$. pyogenes.

Combination of experimentally determined essentiality and GEM based essentiality prediction were shown to be associated to a higher protein persistence than each of them individually. These results are to be expected since In vitro essentiality measurements are often only available for one condition, while GEM can easily be used to predict essentiality over multiple media conditions. Our GEM analysis predicted 153 Staphylococcus aureus genes to be essential in $90 \%$ of the minimal medium combination tested, while 163 genes were found to be essential for growth on rich medium and minimal medium. For Streptococcus, 196 genes were found to be essential in $90 \%$ of the minimal medium combinations tested, while no genes were found to be essential on rich medium. The Streptococcus model contains exchange reactions for all nutrients necessary for growth, meaning only the biomass reaction was found to be essential. Since we know from experimental results that there are several essential genes in $S$. pyogenes, we chose our method of testing all minimal medium compounds to best balance false positive and false negative results while keeping a unified method for our GEM essentiality analysis in both S. aureus and S. pyogenes. Similar to what has been 
experimentally observed and was shown by previous published GEM simulations [8], our simulations show that Staphylococcus can use amino acids as alternative carbon source for survival in the host [51]. A possible limitation of this approach is that GEM predictions can only be made for metabolic (and their associated) proteins. Although many highly persistent genes tend to be essential, not all are highly persistent. This indicated alternatives in essentiality exist [52]. Similarly, many nonessential genes do have a high persistence, indicating they might be essential for Staphylococcus or Streptococcus specific functions such as survival and growth in non-lab conditions such as those found in the host.

Differences in pathogenesis, essentiality as well as other properties such as drug resistance, arise from different selection pressures for individual species within genera [53]. For example, similar to what was found in this study, a recent study shows that although streptococcal virulence factors have no clear patterns among species groups, some virulence factors were shown to be congruous with the evolution of species groups [13]. Core genes together with accessory genes form a complex network that comprise the molecular basis of virulence in Staphylococcus and Streptococcus [54, 55]. Within some individual species, strong selective pressure exist as was shown for $S$. aureus MRSA resistant species where there is an interplay of two strong evolutionary selective pressures: 1) the host type and 2) the antibiotics used in treatment which varies between humans, pets and livestock [56, 57].

We compared the similarity and differences between Staphylococcus and Streptococcus based on the clustering of species in GO functional trees. Some notable differences where observed between Staphylococcus and Streptoccus. Many 'modification of host morphology' proteins in Staphylococcus are also associated to the GO term 'pathogenesis' while many 'biological adhesion' proteins in Streptococcus are associated to the GO term 'pathogenesis'. These results could indicate that modification of host morphology is important for the pathology of Staphylococcus strains while biological adhesion is more important for the pathology of Streptococcus.

Next, we looked at which pathogenesis proteins separate Staphylococcus from Streptococcus species. Analysis of the presence and absence of proteins associated to 'pathogenesis' reveals that Staphylococcus sciuri GCA:002072755 and Staphylococcus haemolytic us GCA:001611955 only contain one pathogenesis protein (PF04647) that is not present in any Streptococcus strain. These results could indicate that horizontal gene transfer of pathogenic proteins occurred between Staphylococcus and Streptococcus or that they only carry pathogenesis proteins derived from a common ancestor.
Additionally, some pathogenic proteins only occur in one or a few genomes, indicating horizontal gene transfer from species outside the Staphylococcus and Streptococcus genus. Horizontal gene transfer is known to be a driving factor in the development of pathogenesis in Staphylococcus and Streptococcus [2, 6, 58-60]. For example, fibronectin binding domain PF02986 has been acquired by Staphylococcus and Streptococcus from an animal host, further spread among different Streptococci and Staphylococci through horizontal gene transfer, and further evolved through domain shuffling [41, 61].

Clustering of Streptococcus and Staphylococcus species based on different GO functional groups revealed sub cluster to be present for S. suis based on GO functional groups 'modification of host morphorlogy' and 'pathogenesis', and revealed a sub cluster to be present for S. agalactiae based on GO functional groups 'biological adhesion' and 'pathogenesis'. What is more, these sub cluster coincides with the potential to infect multiple hosts. It is known that predominantly S. suis serotype 2 strains are associated to zoonotic potential [62, 63]. However, as we could see in Fig. 6a-c, serotype information is not able to separate zoonotic and non-zoonotic $S$. suis strains.

Based on their isolation host we can see that the first group are S. suis strains are zoonotic, while the second group are non-zoonotic strains. Furthermore, human infections with strains for all serotypes in the first cluster have been reported [64, 65]: these results show that these functional groups of proteins can be used to predict the $S$. suis zoonotic potential. Interestingly, the zoonotic group of $S$. suis strains clusters with the human and dog oral commensal $S$. intermedius which can cause meningitis through brain abscesses as well as liver abscesses and in some rare cases endocarditis [66]. Since $S$. suis and S. intermedius are distantly related, this clustering is specific for proteins with functions in modification of host morphology and pathogenesis.

The similarity of phenotypes such as causing meningitis and tropism for brain and liver, suggests these traits may be caused by 'modification of morphology or physiology of other organism' and 'pathogenesis' proteins, and suggest a causal relationship between the proteins associated to these GO terms and the observed phenotype.

Investigation of proteins required to predict $S$. suis zoonotic potential using a random forest classifier revealed PF01289 thiol-activated cytolysin, 2) PF17440 a thiol-activated cytolysin beta sandwich domain to be the two most important factors associated to zoonotic potential. In support of these findings, it was found that a S. suis cytolysin knockout mutant made the strain nonhaemolytic and non-cytotoxic for cultured macrophagelike cells [67] while increased secretion of thiol activated cytolysins was shown to directly cause epithelial cell 
damage in humans, allowing S. suis to spread into deeper tissues [68]. Based on these studies it appears these two cytolysins are involved in formation of a poreforming complex in cholesterol containing host membranes, which explains their importance for conferring zoonotic potential.

It has been suggested that $S$. agalactiae may have jumped from animals to humans in a certain moment of the evolution although it is still debatable if this zoonotic potential remains nowadays [69]. Here, we show that based on their genomic content $S$. agalactiae can be separated in two groups, one that is zoonotic and infects humans, fish and dog, and one group that only infects fish. This separation can be made based on all proteins, indicating zoonotic and non-zoonotic species are likely to have separated some time ago. In the $t$-SNE plots of biological adhesion and pathogenesis a third group can be seen of strains that infects mainly fish but also cow and human. This third cluster contains S. agalactiae strains that infect human most likely originate from this cluster and have further adapted to their human host by acquisition of proteins involved in biological adhesion and pathogenesis. The strains in this group appear to retain zoonotic potential since the cluster contains isolates from humans, fish and dog.

Investigation of proteins required to predict $S$. agalactiae zoonotic potential using a random forest classifier revealed multiple proteins to be important for classifying species as zoonotic. S. agalactiae 5'-nucleotidase-C is present in two proteins: Trifunctional nucleotide phospho-esterase protein YfkN precursor and Endonuclease YhcR precursor. Secreted nucleases play a role in evasion of the human innate immune response via destruction of extracellular traps and interference with phagocytosis signals [70]. Fibrinogen binding protein A allows $S$. agalactiae to attach to fibrinogen and to aggregate platelets [71]. Rib protein contains a Rib domain that confers protective immunity and an alpha $\mathrm{C}$ and alpha $\mathrm{N}$ protein domains involved in invasion and translocation along human epithelial cells according to their PFAM description. The pilus complex contains Pillin D1, Pillin B and Pillin D3 domains and contributes to the initial attachment and invasion of lung and cervical epithelial cells [71]. PF02225;PF07554 CspA serine protease breaks down three chemokines that attract and activate neutrophils [72].

In summary, all proteins important for classification of zoonotic potential appear to be causal to the zoonotic potential phenotype. Of these proteins, nucleosidases YfkN, YhcR and fibrinogen binding protein appear to be the most important factors for S. agalactiae zoonotic potential.

\section{Conclusions}

In this study we dissected Staphylococcus and Streptococcus pathogenesis through the systematic and integrated analysis of genomic, functional, metabolic, and expression data. Both genera were found to have a closed pangenome and lower expression variation for essential and highly persistent genes than for non-essential and low persistent genes.

The study of functional groups of proteins in the pangenome of Staphylococcus and Streptococcus involved in pathogenesis, indicates that domain shuffling and horizontal gene transfer have played an important role in the development and acquisition of pathogenesis proteins of Staphylococcus and Streptococcus species.

The analysis of bacterial clusters based on functional groups of proteins involved in pathogenesis shows that clustering of strains correlates with phenotypes such as zoonotic potential.

Comparison between S. aureus and S. pyogenes indicate three proteins, Enterotoxin B C-terminal domain, Enterotoxin B N-terminal beta-grasp domain together with several functionally equivalent proteins allow Staphylococcus aureus and Streptococcus pyogenes to cause necrotizing fasciitis.

We have also shown that prediction of the phenotype zoonotic potential only requires information about a few proteins, suggesting a direct causal relationship with zoonotic potential.

These findings will enable further research in each of the areas addressed, whereas the approaches and methods herein deployed provide a solid basis towards large-scale prediction of phenotypes based on genomic information.

\section{Methods}

\section{Genome retrieval and annotation}

All available completely assembled genomes of 235 Staphylococcus and 315 Streptococcus were downloaded as EMBL files from EBI-ENA using the Python EnaBrowserTool [73]. Lists of these genomes accession number, name and taxon ID can be found in supplementary material [Additional file 1 and 2]. Genome EMBL files were converted to RDF and de novo annotation was performed storing the results in a graph file per genome using SAPP, a Semantic Annotation Platform with Provenance [74] and the GBOL ontology [75]. Gene calling was performed using Prodigal with codon Table 5 [76]. Annotation was performed using InterProScan version 5.25 [77]. Protein domains were identified by InterProScan by their Pfam identifier [78]. The GNU "parallel" package was used to perform all of the above steps in parallel [79].

The graph files were loaded in GraphDB Free version 8.4.1 in order to query the annotated genomes. Additionally, taxonomic information from UniProt was downloaded in RDF format and loaded in GraphDB. The GraphDB SPARQL endpoint was queried using the 
Table 5 Gene Ontology (GO) terms used to select proteins based on their domain content for functional trees, PCA and tSNA analysis. GO terms that are direct children of the 'Biological process' GO term are marked with an asterisk $\left(^{*}\right)$

\begin{tabular}{ll}
\hline GO ID & Description \\
\hline GO:0008150 & Biological process \\
GO:0008152 & *Metabolic process \\
GO:0017144 & Drug metabolic process \\
GO:0042493 & Response to drug \\
GO:0023052 & *Signalling \\
GO:0065007 & *Biological regulation \\
GO:0022610 & *Biological adhesion \\
GO:0044406 & Adhesion of symbiont to host \\
GO:0051704 & Multi-organism process \\
GO:0044419 & Inter species interaction between organisms \\
GO:0042710 & Biofilm formation \\
GO:0098743 & Cell aggregation \\
GO:0044403 & Symbiont process \\
GO:0009372 & Quorum sensing \\
GO:0035821 & Modification of morphology or physiology \\
GO:0009405 & of other organism \\
\hline
\end{tabular}

Python SPARQLWrapper [80] package and the R Curl package [81] to retrieve information and store them as matrixes given in supplementary material [see Additional file 3 and 4]. These files were used for all subsequent analyses.

\section{Estimation of the size of the pan- and core genome} Proteins were compared based on their Pfam domain content. We defined protein domain content as the alphabetical order of all unique domains associated with a given protein. A matrix was built to collect information on the presence or absence of proteins in each genome. Two sampling approaches were used: 1) genomes were randomly selected from all genomes in the analysed genera and 2) a maximum of one genome per species was selected to avoid bias introduced by species with many sequenced genomes. One up to the total number of genomes were sampled and analysed using the micropan $\mathrm{R}$ package [24] to investigate the effect of the number of genomes on the estimation of the size of the pan- and core genome. Additionally, these samples we used to estimate the sizes of the pan- and core genome using a binomial mixture model using the micropan BinomixEstimate function with 5000 permutations and a core detect probability of 1 . The process was repeated 10 times to estimate the variance of the estimated size of the panand core genomes. The Heaps' function was used to fit a
Heaps' regression model; $\alpha>1$ indicates convergence of the size of the pan-genome and that it is closed.

\section{Variability of gene expression and its association to persistence}

Gene variability was calculated based on $156 \mathrm{~S}$. aureus RNA samples from 44 conditions ranging from laboratory to conditions mimicking infection, measured by Tiling arrays [42]. These 44 conditions can be categorized in four groups:

1) rich medium (TSB), 2) minimal medium (CDM), 3) cell culture media (RPMI, pMEM) 4) in human plasma (plasma), 5) growth with human bronchial epithelial cell line S9 and the human monocyte cell line THP-1.

Samples were taken at different time points and for infection simulations oxygen availability was limited at later time points. For a complete description of the conditions we refer to S1 Data in the original paper by $U$. Mader et al. [42]. For every gene we considered its expression profile over all samples and a variability value was calculated as the ratio between the standard deviation and the mean expression value using the same approach as in Koehorst et al. [9].

\section{Protein persistence and essentiality}

We defined the persistence of a gene as

$$
\text { Persistence }=\frac{N(\text { orth })}{N}
$$

where $N$ (orth) is the number of genomes carrying a given orthologue and $N$ is the number of genomes searched [82]. Orthologue genes were identified as genes with identical protein domain content. Locus tags associated to the genes were inferred from the original annotation and used to integrated genome wide gene essentiality data from transposon mutagenesis studies for Staphylococcus strains S0385 grown on whole porcine blood [83], NCTC8325 Newman grown on BHI broth [43] and JE2 grown on Handke mannitol medium [84] and Streptococcus strains S. pyogenes M1T1 strain 5448 and M49 strain NZ131 grown in rich Todd-Hewitt Yeast (THY) medium [85].

\section{GEM-based predictions of essentiality}

Gene essentiality analysis based on genome scale modelling was performed using the genome-scale, constraintbased metabolic model (GEM) of $S$. aureus NTCTC 8325 [8] and the GEM model of S. pyogenes M49 [86]. First, a minimal medium was determined using the 'cobrapy minimal_medium function'. All carbon, nitrogen, sulphur and phosphor sources from the medium that could support growth were detected by substituting the default carbon, nitrogen, sulphur and phosphor 
sources. All combinations of minimal media containing these carbon, nitrogen, sulphur and phosphor sources were generated.

Gene essentiality for all combinations of minimal media containing these carbon, nitrogen, sulphur and phosphor were tested by performing single gene deletions followed by flux balance analysis optimizing for growth. If a gene knock-out reduced predicted growth for the media compositions below $1 \%$ the gene was considered conditionally essential. Genes predicted to be conditionally essential in at least $90 \%$ of the in-silico media compositions were marked as essential.

All optimizations were performed using the Gurobi optimizer 8.1 [87] with COBRApy 0.13.4 [88] and Python 3.6.

\section{Functional analysis}

Genome information was retrieved from associated literature and from the Biosample database [89], including serotype information and zoonotic potential and isolation-host. Zoonotic classification was derived from literature for S. inae [90-93], S. agalactiae [69], S. dysgalactiae, S. equi [94-96] and at the serotype level for $S$. suis [97-99]. For all zoonotic Streptococcus species data about the isolation host was retrieved from the Biosamples databases [100] or literature [63-65, 101-108]. Additional Gene Ontology (GO) annotation from the GODM (GO Domain Miner) database [33] was added to proteins based on their domain content, increasing the number of GO terms by approximately 10-fold compared to GO term annotation retrieved from the InterPro database. Literature was used to select 17 GO terms in the Biological process ontology with known or suspected association to pathogenesis [109-113] (Table 5).

The presence/absence matrix of proteins was filtered on proteins annotated with any of the 17 GO terms (Table 5) or their descendent GO terms using the $\mathrm{R}$ GO.db package [114]. The filtered matrix was used to calculate the Euclidean distance between genomes. Hierarchical complete-linkage clustering was used to generate dendrograms. These GO-specific dendrograms were compared to a reference dendrogram based on all proteins.

Because these dendrograms are based on annotation of proteins for a specific function, we will refer to them as 'functional trees'. Euclidean distances of genomes in the functional trees and the reference tree were calculated and scaled to values between 0 and 1 using the $R$ scale function using the minimum value for centring, and (min - max) for scaling. Scaled values were used to calculate similarity scores for the position of genomes in each functional tree compared to the reference. These similarity scores were calculated as the Pearson correlation between the scaled Euclidean distances of genomes in the functional tree and the scaled Euclidean distance in the reference tree. Interactive heatmap were generated showing the presence and absence of proteins per genome, while showing the similarity in the side column to highlight differences compared to the reference tree. These interactive graphs were generated using the dendextend and heatmaply packages $[115,116]$. Similarity scores for functional trees were calculated using the dendextend cor_cophenetic function.

Matrix manipulations, Principal Component Analysis (PCA), t-Distributed Stochastic Neighbour Embedding (t-SNE) and graphs were performed using R 3.6.1 [117], the prcomp command, and the Rtsne 0.15 [118] and ggplot2 2.3.2.1 [119] packages. t-SNE was performed with default parameters.

\section{Random Forest classification}

Proteins belonging to GO categories 'pathogenesis', 'modification of morphology or physiology of other organisms' and 'biological adhesions', were used to train Random Forests classifiers for S. suis and S. agalactiae strains to predict whether they belong to the class 'zoonotic potential' on 'non-zoonotic potential'. This classification was based on the clustering of in PCA and t-SNE plots which revealed the presence of a zoonotic and a non-zoonotic group of strains. Data was split in $75 \%$ training data and $25 \%$ validation data.

Data was loaded using Python 3.6, pandas 0.24.2. Skicit-learn 0.20.3 used to load data and train Random Forest classifiers. Treeinterpreter 0.1.0 was used to interpret feature (protein) importance for classification in general and feature contribution to predict specific classes. Grid search for 300 combinations of parameters was performed optimizing the parameters $\mathrm{n}$-estimators, max_features, max_depth, min_samples_split, and the min_samples. Iterative rounds of feature reduction, that is removal of the protein which least contribute to the classification, followed by hyper parameter optimization, was used to find the minimal set of features (proteins) needed to classify both training and test data. Feature importance and contribution were plotted using matplotlib 3.0.3.

\section{Supplementary Information}

The online version contains supplementary material available at https://doi. org/10.1186/s12864-021-07388-6.

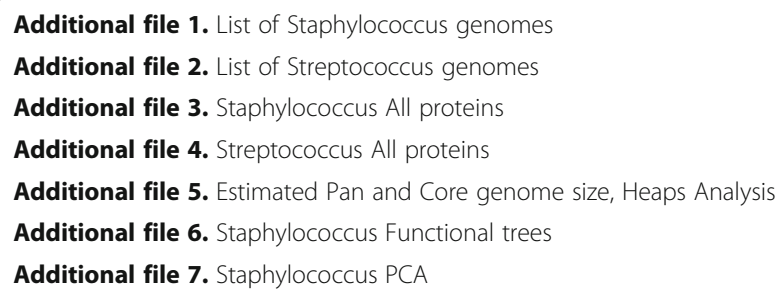



Additional file 8. Staphylococcus t-SNE
Additional file 9. Streptococcus Functional trees
Additional file 10. Streptococcus PCA
Additional file 11. Streptococcus t-SNE
Additional file 12. Staphylococcus \& Streptococcus combined PCA
Additional file 13. Staphylococcus \& Streptococcus combined t-SNE
Additional file 14. Optimal hyper parameters

\section{Abbreviations}

Exp: Experimentally; GEM: Genome scale metabolic model; GO: Genome Ontology; IgA: Immunoglobulin A; IgG: Immunoglobulin G; t-SNE: Tdistributed Stochastic Neighbour Embedding

\section{Acknowledgements}

We would like to thank Jianan Chen for performing the initial data exploration for the genome comparison of Staphylococcus and Streptococcus.

\section{Authors' contributions}

NZ performed the main analyses and wrote the draft manuscript. MSD and ES participated in the design of the study and supervised and directed the research. MSD and ES and VDMS revised the manuscript. All authors contributed to the writing of the final version of the manuscript. The authors read and approved the final manuscript.

\section{Funding}

This work has been supported by European Union through the FP7 programme under grant agreement No. 305340 (INFECT), the SysteMTb project (HEALTH-F4-2010-241,587) and the Horizon 2020 research and innovation programme under grant agreement No. 634942 (MycoSynVac) and from The Netherlands Organisation for Health Research and Development (ZonMW) through the PERMIT project (Personalized Medicine in Infections: from Systems Biomedicine and Immunometabolism to Precision Diagnosis and Stratification Permitting Individualized Therapies, project number 456008002) under the PerMed Joint Transnational call JTC 2018 (Research projects on personalised medicine - smart combination of pre-clinical and clinical research with data and ICT solutions). The funders had no role in study design, data collection and analysis, decision to publish, or preparation of the manuscript.

\section{Availability of data and materials}

The authors declare that all data supporting the findings of this study are available within the article and its supplementary information.

\section{Ethics approval and consent to participate}

Not applicable.

\section{Consent for publication}

Not applicable.

\section{Competing interests}

The authors declare that they have no competing interests.

\section{Author details}

${ }^{1}$ Laboratory of Systems and Synthetic Biology, Wageningen University \& Research, Stippeneng 4, 6708WE Wageningen, Netherlands. ${ }^{2}$ LifeGlimmer GmBH, Markelstraße 38, 12163, Berlin, Germany.

Received: 1 May 2020 Accepted: 13 January 2021

Published online: 04 February 2021

\section{References}

1. Graham MR, Smoot LM, Migliaccio CAL, Virtaneva K, Sturdevant DE, Porcella $\mathrm{SF}$, et al. Virulence control in group a streptococcus by a two-component gene regulatory system: global expression profiling and in vivo infection modeling. Proc Natl Acad Sci U S A. 2002;99(21):13855-60 Available from: http://www.pnas.org/cgi/content/long/99/21/13855.
2. Krismer B, Weidenmaier C, Zipperer A, Peschel A. The commensal lifestyle of Staphylococcus aureus and its interactions with the nasal microbiota. Nat Rev Microbiol. 2017;15(11):675-87. https://doi.org/10.1038/nrmicro.2017.104.

3. Henriques-Normark B, Normark S. Commensal pathogens, with a focus on Streptococcus pneumoniae, and interactions with the human host. Exp Cell Res. 2010;316(8):1408-14. https://doi.org/10.1016/.yexcr.2010.03.003.

4. Causey WA. Staphylococcal and streptococcal infections of the skin. Prim Care. 1979;6(1):127-39 Available from: http://www.ncbi.nlm.nih.gov/ pubmed/8902344

5. Patenge N, Pappesch R, Khani A, Kreikemeyer B. Genome-wide analyses of small non-coding RNAs in streptococci. Front Genet. 2015;6(MAY):1-13.

6. Suzuki H, Lefébure T, Bitar PP, Stanhope MJ. Comparative genomic analysis of the genus Staphylococcus including Staphylococcus aureus and its newly described sister species Staphylococcus simiae. BMC Genomics. 2012;13(1): 38 Available from: http://www.biomedcentral.com/1471-2164/13/38.

7. Saccenti E, Nieuwenhuijse D, Koehorst JJ, Dos Santos VAPM, Schaap PJ. Assessing the metabolic diversity of streptococcus from a protein domain point of view. PLoS One. 2015;10(9):1-20.

8. Bosi E, Monk JM, Aziz RK, Fondi M, Nizet V, Palsson BØ. Comparative genome-scale modelling of Staphylococcus aureus strains identifies strainspecific metabolic capabilities linked to pathogenicity. Proc Natl Acad Sci. 2016;113(26):E3801-9. https://doi.org/10.1073/pnas.1523199113.

9. Koehorst JJ, Van Dam JCJ, Van Heck RGA, Saccenti E, Dos Santos VAPM, Suarez-Diez M, et al. Comparison of 432 pseudomonas strains through integration of genomic, functional, metabolic and expression data. Sci Rep. 2016;6(December):1-13. https://doi.org/10.1038/srep38699.

10. Koehorst JJ, Saccenti E, Schaap PJ, dos Santos VAP M, Suarez-Diez M. Protein domain architectures provide a fast, efficient and scalable alternative to sequence-based methods for comparative functional genomics. F1000Research. 2017;5(0):1987 Available from: https:/f1000research.com/ articles/5-1987/v3.

11. Rouli L, Merhej V, Fournier PE, Raoult D. The bacterial pangenome as a new tool for analysing pathogenic bacteria. New Microbes New Infect. 2015;7: 72-85. https://doi.org/10.1016/j.nmni.2015.06.005.

12. Fuchs S, Mehlan H, Bernhardt J, Hennig A, Michalik S, Surmann K, et al. AureoWiki-the repository of the Staphylococcus aureus research and annotation community. Int J Med Microbiol. 2018;308(6):558-68. https://doi. org/10.1016/j.jmm.2017.11.011.

13. Gao XY, Zhi XY, Li HW, Klenk HP, Li WJ. Comparative genomics of the bacterial genus streptococcus illuminates evolutionary implications of species groups. PLoS One. 2014;9(6):e101229.

14. Snipen L, Almøy T, Ussery DW. Microbial comparative pan-genomics using binomial mixture models. BMC Genomics. 2009;10:1-8.

15. Tettelin H, Riley D, Cattuto C, Medini D. Comparative genomics: the bacterial pan-genome. Curr Opin Microbiol. 2008;11(5):472-7.

16. Protein F, Jeng A, Sakota V, Li Z, Datta V, Beall B, et al. Molecular genetic analysis of a group a streptococcus operon encoding serum opacity factor and a novel. J Bacteriol. 2003;185(4):1208-17 Available from: http://jb.asm. org/content/185/4/1208.full.pdf.

17. Kuroda M, Ohta T, Uchiyama I, Baba T, Yuzawa H, Kobayashi I, et al. Whole genome sequencing of meticillin-resistant Staphylococcus aureus. The Lancet 2001;357(9264):1225--40. https://doi.org/10.1016/S01406736(00)04403-2.

18. Matyi SA, Dupre JM, Johnson WL, Hoyt PR, White DG, Brody T, et al. Isolation and characterization of Staphylococcus aureus strains from a Paso del Norte dairy. J Diary Sci HHS Public Access. 2017;96(6):3535-42.

19. Staphylococcus TF. Chapter 12 in microbiology. In: S B, editor. Microbiology 4th edition. 4th ed. Galveston: University of Texas Medical Branch at Galveston; 1996. Chapter 12. Available from: https://www.ncbi.nlm.nih.gov/ books/NBK8448/996.

20. Li Y, Cao B, Zhang Y, Zhou J, Yang B, Wang L. Complete genome sequence of Staphylococcus aureus T0131, an ST239-MRSA-SCCmec type III clone isolated in China. J Bacteriol. 2011;193(13):3411-2.

21. Sass P, Berscheid A, Jansen A, Oedenkoven M, Szekat C, Strittmatter A, et al. Genome sequence of Staphylococcus aureus VC40, a vancomycin- and daptomycin-resistant strain, to study the genetics of development of resistance to currently applied last-resort antibiotics. J Bacteriol. 2012;194(8):2107-8.

22. Baba T, Bae T, Schneewind O, Takeuchi F, Hiramatsu K. Genome sequence of Staphylococcus aureus strain Newman and comparative analysis of staphylococcal genomes: polymorphism and evolution of two major pathogenicity islands. J Bacteriol. 2008;190(1):300-10. 
23. Zautner AE, Krause M, Stropahl G, Holtfreter S, Frickmann H, Maletzki C, et al. Intracellular persisting Staphylococcus aureus is the major pathogen in recurrent tonsillitis. PLoS One. 2010;5(3).

24. Surmann K, Depke M, Dhople VM, Pané-Farré J, Hildebrandt P, Gumz J, et al. Analysis of Staphylococcus aureus proteins secreted inside infected human epithelial cells. Int J Med Microbiol. 2018;308(6):664-74. https://doi.org/10. 1016/j.jimm.2018.06.002.

25. Sendi P, Proctor RA. Staphylococcus aureus as an intracellular pathogen: the role of small colony variants. Trends Microbiol. 2009;17(2):54-8.

26. Kreikemeyer B, Mclver KS, Podbielski A. Virulence factor regulation and regulatory networks in streptococcus pyogenes and their impact on pathogen-host interactions. Trends Microbiol. 2003;11(5):224-32.

27. Loof TG, Deicke C, Medina E. The role of coagulation/fibrinolysis during streptococcus pyogenes infection. Front Cell Infect Microbiol. 2014; 4(September):1-8. https://doi.org/10.3389/fcimb.2014.00128/abstract.

28. Askarian F, Ajayi C, Hanssen A, Van Sorge NM. The interaction between Staphylococcus aureus SdrD and desmoglein 1 is important for adhesion to host cells. Nat Publ Gr. 2016;(October 2015):1-11. https://doi.org/10.1038/ srep22134.

29. Eriksen NHR, Espersen F, Rosdahl VT, Jensen K, Godwin H, Wyllie DH, et al. Prevalence of Staphylococcus aureus protein a (spa) mutants in the community and hospitals in Oxfordshire. Epidemiol Infect. 1995;115(01):5160 Available from: http://www.journals.cambridge.org/abstract_S095026 8800058118.

30. Hermans SJ, Baker HM, Sequeira RP, Langley RJ, Baker EN, Fraser JD. Structural and functional properties of staphylococcal superantigen-like protein 4. Infect Immun. 2012;80(11):4004-13.

31. Jenkins A, Diep A, Mai T, Vo NH, Warrener P, Suzich J, et al. Differential expression and roles of Staphylococcus aureus virulence determinants during colonization and disease. MBio. 2015;6(1):1-10.

32. Virulence factors of Streptococcus pyogenes and their roles [Internet]. [cited 2018 Dec 17]. Available from: https://microbeonline.com/virulence-factorsstreptococcus-pyogenes-roles/.

33. Alborzi SZ, Devignes M, Ritchie D, Alborzi SZ, Devignes M, Ritchie D, et al, Associating gene ontology terms with Pfam protein domains to cite this version : HAL id : HAL-01531204 associating gene ontology terms with Pfam protein domains; 2017.

34. Stemberk V, Jones RPO, Moroz O, Atkin KE, Edwards AM, Turkenburg JP, et al. Evidence for steric regulation of fibrinogen binding to Staphylococcus aureus Fibronectin-binding. Protein A. 2014;289(18):12842-51.

35. Fries BC, Varshney AK. Bacterial toxins - staphylococcal enterotoxin B description of agent; 2013. p. 1-12.

36. Zheng X, Bi C, Brooks M, DSH. Collagen-like proteins of pathogenic streptococci. Anal Chem. 2015;25(4):368-79.

37. Rohde M, habi rer nat, Cleary PP. Adhesion and invasion of Streptococcus pyogenes into host cells and clinical relevance of intracellular streptococci. Univ Oklahoma Heal Sci Cent. 2016:1-30.

38. Gottlieb M, Long B, Koyfman A. The evaluation and management of toxic shock syndrome in the emergency department: a review of the literature. J Emerg Med. 2018;(December 2017):1-8. https://doi.org/10.1016/j.jemermed. 2017.12.048

39. Otto M. Staphylococcus aureus toxins Michael. Curr Opin Microbiol. 2015: 32-7.

40. Kolar SL, Antonio Ibarra J, Rivera FE, Mootz JM, Davenport JE, Stevens SM, et al. Extracellular proteases are key mediators of Staphylococcus aureus virulence via the global modulation of virulence-determinant stability. Microbiologyopen. 2013;2(1):18-34

41. Chen WH, Lu G, Chen X, Zhao XM, Bork P. OGEE v2: an update of the online gene essentiality database with special focus on differentially essential genes in human cancer cell lines. Nucleic Acids Res. 2017;45(D1):D940-4

42. Mäder U, Nicolas P, Depke M, Pané-Farré J, Debarbouille M, van der Kooi-Pol MM, et al. Staphylococcus aureus Transcriptome architecture: from laboratory to infection-mimicking conditions. PLoS Genet. 2016; 12(4):1-32.

43. Chaudhuri RR, Allen AG, Owen PJ, Shalom G, Stone K, Harrison M, et al. Comprehensive identification of essential Staphylococcus aureus genes using transposon-mediated differential hybridisation (TMDH). BMC Genomics. 2009;10:1-18.

44. Forsyth RA, Haselbeck RJ, Ohlsen KL, Yamamoto RT, Xu H, Trawick JD, et al. A genome-wide strategy for the identification of essential genes in Staphylococcus aureus. Mol Microbiol. 2002;43(6):1387-400.
45. Henderson B, Nair S, Pallas J, Williams MA. Fibronectin: a multidomain host adhesin targeted by bacterial fibronectin-binding proteins. FEMS Microbio Rev. 2011;35(1):147-200.

46. Edwards AM, Potts JR, Josefsson E, Massey RC. Staphylococcus aureus host cell invasion and virulence in sepsis is facilitated by the multiple repeats within FnBPA. PLoS Pathog. 2010;6(6):e1000964. https://doi.org/10.1371/ journal.ppat.1000964.

47. Timmer AM, Kristian SA, Datta V, Jeng A, Gillen CM, Walker MJ, et al. Serum opacity factor promotes group A streptococcal epithelial cell invasion and virulence. Mol Microbiol. 2006;62(1):15-25 Available from: http://www. embase.com/search/results?subaction=viewrecord\&from $=$ export\&id $=\llcorner 443$ 86547\%5Cn, https://doi.org/10.1111/j.1365-2958.2006.05337.x, http://munprimo.hosted.exlibrisgroup.com/openurl/01MUN/01MUN_SERVICES?sid= EMBASE\&issn=0950382X\&id=doi:10.1111\%2Fj.

48. Spaulding AR, Salgado-Pabón W, Kohler PL, Horswill AR, Leung DYM, Schlievert PM. Staphylococcal and streptococcal superantigen exotoxins. Clin Microbiol Rev. 2013;26(3):422-47.

49. Klingelhutz AJ, Salgado-pabón W. The Superantigen toxic shock syndrome toxin 1 alters human aortic endothelial cell function. Infect Immun. 2018; 86(3):1-16. https://doi.org/10.1128/IAl.00848.17.

50. Stoll H, Ost M, Singh A, Mehling R, Neri D, Schäfer I, et al. Staphylococcal enterotoxins dose-dependently modulate the generation of myeloidderived suppressor cells. Front Cell Infect Microbiol. 2018:8(September):1-15.

51. Bae T, Banger AK, Wallace A, Glass EM, Aslund F, Schneewind O, et al. Staphylococcus aureus virulence genes identified by bursa aurealis mutagenesis and nematode killing. Proc Natl Acad Sci. 2004;101(33):123127.

52. Kamminga T, Koehorst JJ, Vermeij P, Slagman S-J, Martins dos Santos VAP, Bijlsma JJE, et al. Persistence of functional protein domains in mycoplasma species and their role in host specificity and synthetic minimal life. Front Cell Infect Microbiol. 2017;7:31 [cited 2017 Feb 7]. https://doi.org/10.3389/ fcimb.2017.00031/full.

53. Cooper VS, Honsa E, Rowe H, Deitrick C, Iverson AR, Whittall JJ, et al. Experimental evolution in vivo to identify selective pressures during pneumococcal colonization. bioRxiv. 2020;5(3):1-17.

54. Pain M, Hjerde E, Klingenberg C, Cavanagh JP. Comparative genomic analysis of Staphylococcus haemolyticus reveals key to hospital adaptation and pathogenicity. Front Microbiol. 2019;10(September):1-13.

55. Anisimova M, Bielawski J, Dunn K, Yang Z. Phylogenomic analysis of natural selection pressure in streptococcus genomes. BMC Evol Biol. 2007;7:1-13.

56. Jamrozy D, Coll F, Mather AE, Harris SR, Harrison EM, MacGowan A, et al. Evolution of mobile genetic element composition in an epidemic methicillin-resistant Staphylococcus aureus: temporal changes correlated with frequent loss and gain events. BMC Genomics. 2017;18(1):1-12.

57. Mccarthy AJ, Lindsay JA, Loeffler A. Are all meticillin-resistant Staphylococcus aureus (MRSA) equal in all hosts? Epidemiological and genetic comparison between animal and human MRSA. Vet Dermatol. 2012;23(4):267-75.

58. Osaki M, Takamatsu D, Shimoji Y, Sekizaki T. Characterization of Streptococcus suis genes encoding proteins homologous to sortase of grampositive bacteria. J Bacteriol. 2002;184(4):971-82 Available from: http://www. pubmedcentral.nih.gov/articlerender.fcgi?artid=134807\&tool= pmcentrez\&rendertype=abstract.

59. Kao C, Chen P, Huang F, Chen C, Chi C, Lin Y, et al. Clinical and genetic analysis of invasive and non-invasive group A streptococcal infections in Central Taiwan; 2005. p. 105-11.

60. McNeilly CL, McMillan DJ. Horizontal gene transfer and recombination in Streptococcus dysgalactiae subsp. equisimilis. Front Microbiol. 2014;5(DEC): $1-6$.

61. Bork P, Doolittle RF. Proposed acquisition of an animal protein domain by bacteria. Proc Natl Acad Sci. 1992;89(19):8990-4. https://doi.org/10.1073/ pnas.89.19.8990.

62. Goyette-Desjardins G, Auger JP, Xu J, Segura M, Gottschalk M. Streptococcus suis, an important pig pathogen and emerging zoonotic agent-an update on the worldwide distribution based on serotyping and sequence typing. Emerg Microbes Infect. 2014;3(March):e45.

63. Kerdsin A, Puangpatra P, Tanimura S, Gottschalk M, Polwichai P, Dejsirilert S, et al. Genotypic profile of Streptococcus suis serotype 2 and clinical features of infection in humans, Thailand. Emerg Infect Dis. 2011;17(5):835-42.

64. Nghia HDT, Ngo TH, Le DL, Campbell J, To SD, Chau NW, et al. Human case of Streptococcus suis serotype 16 infection. Emerg Infect Dis. 2008:14(1): 155-7. 
65. Wertheim HFL, Nghia HDT, Taylor W, Schultsz C. Streptococcus suis: an emerging human pathogen. Clin Infect Dis. 2009;48(5):617-25.

66. Hasegawa N, Sekizuka T, Sugi Y, Kawakami N, Ogasawara Y, Kato K, et al. Characterization of the pathogenicity of Streptococcus intermedius TYG1620 isolated from a human brain abscess based on the complete genome sequence with Transcriptome analysis and transposon mutagenesis in a murine subcutaneous abscess model. Infect Immun. 2017;85(2):1-15.

67. Allen AG, Bolitho S, Lindsay H, Khan S, Bryant C, Norton P, et al. Generation and characterization of a defined mutant of streptococcus suis lacking suilysin. Infect Immun. 2001;69(4):2732-5.

68. He Z, Pian Y, Ren Z, Bi L, Yuan Y, Zheng Y, et al. Increased production of suilysin contributes to invasive infection of the Streptococcus suis strain 05ZYH33. Mol Med Rep. 2014;10(6):2819-26.

69. Botelho ACN, Ferreira AFM, Fracalanzza SEL, Teixeira LM, Pinto TCA. A perspective on the potential zoonotic role of Streptococcus agalactiae: searching for a missing link in alternative transmission routes. Front Microbiol. 2018;9(March):1-5.

70. Remmington A, Turner CE. The DNases of pathogenic lancefield streptococci. Microbiology. 2018;164(3):242-50.

71. Sharma P, Lata H, Arya DK, Kashyap AK, Kumar H, Dua M, et al. Role of pilus proteins in adherence and invasion of streptococcus agalactiae to the lung and cervical epithelial cells. J Biol Chem. 2013; 288(6):4023-34.

72. Bryan JD, Shelver DW. Streptococcus agalactiae CspA is a serine protease that inactivates chemokines. J Bacteriol. 2009:191(6):1847-54.

73. European Bioinformatics Institute. EnaBroswerTools [Internet]. [cited 2019 Nov 7]. Available from: https://github.com/enasequence/ enaBrowserTools.

74. Koehorst JJ, van Dam JCJ, Saccenti E, dos Santos VAP M, Suarez-Diez M, Schaap PJ. SAPP: functional genome annotation and analysis through a semantic framework using FAIR principles. Bioinformatics. 2017;(March):1-3. https://doi.org/10.1093/bioinformatics/btx767/4653704.

75. van Dam JCJ, Koehorst JJ, Vik JO, VAP MDS, Schaap PJ, Suarez-Diez M. The Empusa code generator and its application to GBOL, an extendable ontology for genome annotation. Sci Data. 2019;6(1):254. https://doi.org/10. 1038/s41597-019-0263-7.

76. Hyatt D, Chen GL, LoCascio PF, Land ML, Larimer FW, Hauser LJ. Prodigal: prokaryotic gene recognition and translation initiation site identification. BMC Bioinformatics. 2010;11:119.

77. Finn RD, Attwood TK, Babbitt PC, Bateman A, Bork P, Bridge AJ, et al. InterPro in 2017-beyond protein family and domain annotations. Nucleic Acids Res. 2017:45(D1):D190-9.

78. Finn RD, Coggill P, Eberhardt RY, Eddy SR, Mistry J, Mitchell AL, et al. The Pfam protein families database: towards a more sustainable future. Nucleic Acids Res. 2016;44(D1):D279-85.

79. Tange O. Gnu parallel-the command-line power tool.; login: The USENIX Magazine, 2011;36(1):42-47.

80. SPARQL endpoint interface to python [Internet]. [cited 2018 Aug 8]. Available from: https://rdflib.github.io/sparqlwrapper/.

81. Duncan Temple Lang and the CRAN team. CRAN - Package RCurl [Internet]. [cited 2018 Nov 1]. Available from: https://cran.r-project.org/web/packages/ RCurl/index.html

82. Fang G, Rocha E, Danchin A. How essential are nonessential genes ? 2004.

83. Christiansen MT, Kaas RS, Chaudhuri RR, Holmes MA, Hasman H, Aarestrup FM. Genome-wide high-throughput screening to investigate essential genes involved in methicillin-resistant Staphylococcus aureus sequence type 398 survival. PLoS One. 2014;9(2):e89018.

84. Fey PD, Endres JL, Yajjala VK, Fey PD, Endres $\lrcorner$, Yajjala K, et al. A genetic resource for rapid and comprehensive phenotype. MBio. 2013;4(1):1-8

85. Le Breton Y, Belew AT, Valdes KM, Islam E, Curry P, Tettelin H, et al. Essential genes in the core genome of the human pathogen streptococcus pyogenes. Sci Rep. 2015;5:9838

86. Levering J, Fiedler T, Sieg A, van Grinsven KWA, Hering S, Veith N, et al. Genome-scale reconstruction of the streptococcus pyogenes M49 metabolic network reveals growth requirements and indicates potential drug targets. J Biotechnol. 2016;232:25-37. https://doi.org/10.1016/j.jbiotec.2016.01.035.

87. Gurobi Optimization LLC. Gurobi optimizer reference manual [internet]; 2018. Available from: http://www.gurobi.com.

88. Ebrahim A, JALBO P, Hyduke DR. COBRApy: constraints-based reconstruction and analysis for python. BMC Syst Biol. 2013;7:74. https://doi.org/10.1186/ 1752-0509-7-74.
89. Gostev M, Faulconbridge A, Brandizi M, Fernandez-Banet J, Sarkans U, Brazma A, et al. The BioSample database (BioSD) at the European bioinformatics institute. Nucleic Acids Res. 2012;40(D1):64-70.

90. Zhang BC, Zhang J, Sun L. Streptococcus iniae SF1: complete genome sequence, proteomic profile, and immunoprotective antigens. PLoS One. 2014;9(3):e91324.

91. Pridgeon JW, Zhang D, Zhang L. Complete genome sequence of the attenuated Novobiocin-resistant streptococcus iniae vaccine strain ISNO. Genome Announc. 2014;2(3):2007-8.

92. Sun JR, Yan JC, Yen CY, Lee SY, Lu JJ. Invasive infection with streptococcus iniae in Taiwan. J Med Microbiol. 2007;56(9):1246-9.

93. Rajoo S, Jeon W, Park K, Yoo S, Yoon I, Lee H, et al. Complete genome sequence of streptococcus iniae YSFST01-82, isolated from olive flounder in Jeju, South Korea. Genome Announc. 2015;3(2):10-1.

94. Holden MTG, Heather Z, Paillot R, Steward KF, Webb K, Ainslie F, et al. Genomic evidence for the evolution of Streptococcus equi host restriction, increased virulence, and genetic exchange with human pathogens. PLoS Pathog. 2009;5(3):e1000346.

95. Pelkonen S, Lindahl SB, Suomala P, Karhukorpi J, Vuorinen S, Koivula I, et al. Transmission of streptococcus equi subspecies zooepidemicus infection from horses to humans. Emerg Infect Dis. 2013;19(7):1041-8.

96. Ma Z, Geng J, Zhang H, Yu H, Yi L, Lei M, et al. Complete genome sequence of Streptococcus equi subsp. Zooepidemicus strain ATCC 35246. J Bacteriol. 2011;193(19):5583-4.

97. Zhang Y, Ding D, Liu M, Yang X, Zong B, Wang X, et al. Effect of the glycosyltransferases on the capsular polysaccharide synthesis of Streptococcus suis serotype 2. Microbiol Res. 2016;185:45-54. https://doi. org/10.1016/j.micres.2016.02.002

98. Zhang $Y$, Lu C, Dong W, Pan Z, Zhong X, Lu P, et al. SssP1, a Streptococcus suis fimbria-like Protein transported by the SecY2/A2 system, contributes to bacterial virulence. Appl Environ Microbiol. 2018;84(18):1-17.

99. Chen C, Tang J, Dong W, Wang C, Feng Y, Wang J, et al. A glimpse of streptococcal toxic shock syndrome from comparative genomics of $\mathrm{S}$. suis 2 Chinese isolates. PLoS One. 2007;2(3):e315.

100. Barrett T, Clark K, Gevorgyan R, Gorelenkov V, Gribov E, KarschMizrachi I, et al. BioProject and BioSample databases at NCBI: facilitating capture and organization of metadata. Nucleic Acids Res. 2012;40(D1):57-63.

101. Wang K, Chen J, Yao H, Lu C. Whole-genome sequence of Streptococcus suis serotype 4 reference strain 6407. Genome Announc. 2014;2(4):9-10.

102. Pan Z, Ma J, Dong W, Song W, Wang K, Lu C, et al. Novel variant serotype of Streptococcus suis isolated from piglets with meningitis. Appl Environ Microbiol. 2015;81(3):976-85.

103. Xiao J, Hu P, Chen H, Wu J, Hua Y, Zhang A, et al. Comparative genomic analysis of Streptococcus suis reveals significant genomic diversity among different serotypes. BMC Genomics. 2011;12(1):523 Available from: http:// www.biomedcentral.com/1471-2164/12/523.

104. Chatellier S, Harel J, Zhang Y, Higgins R, Brousseau R, Gottschalk M, et al. Phylogenetic diversity of Streptococcus suis strains of various serotypes as revealed by 165 rRNA gene sequence comparison. Int J Syst Bacteriol. 2009; 48(2):581-9

105. Pan $X$, Tang J, Zhao Y, Li M, Yao X, Hu D, et al. Isolation and characterization of a native avirulent strain of Streptococcus suis serotype 2: a perspective for vaccine development. Sci Rep. 2015;5(1):1-8.

106. Boyle B, Vaillancourt K, Bonifait L, Charette SJ, Gottschalk M, Grenier D. Genome sequence of the swine pathogen streptococcus suis serotype 2 strain S735. J Bacteriol. 2012;194(22):6343-4.

107. Wang K, Yao H, Chengping Lu JC. Complete genome sequence of Streptococcus suis serotype 16 strain TL13. J Bacteriol. 2011;193(9): 2375-6.

108. Zheng H, Du P, Qiu X, Kerdsin A, Roy D, Bai X, et al. Genomic comparisons of Streptococcus suis serotype 9 strains recovered from diseased pigs in Spain and Canada. Vet Res. 2018;49(1):1-13. https://doi.org/10.1186/s13567017-0498-2.

109. Szafrański SP, Deng ZL, Tomasch J, Jarek M, Bhuju S, Rohde M, et al. Quorum sensing of Streptococcus mutans is activated by Aggregatibacter actinomycetemcomitans and by the periodontal microbiome. BMC Genomics. 2017;18(1):1-15.

110. Cook LC, LaSarre B, Federle MJ. Interspecies communication among commensal and pathogenic streptococci. MBio. 2013;4(4):1-11. 
111. Brouwer S, Cork AJ, Ong C-LY, Barnett TC, West NP, Mclver KS, et al. The endopeptidase PepO regulates the SpeB cysteine protease and is essential for the virulence of invasive M1T1 Streptococcus pyogenes. J Bacteriol. 2018; (January):JB.00654-17 10.1128/JB.00654-17.

112. Cheung GYC, Otto M. Understanding the significance of Staphylococcus epidermidis bacteremia in babies and children. Curr Opin Infect Dis. 2010; 23(3):208-16.

113. Herman-Bausier P, Labate C, Towell AM, Derclaye S, Geoghegan JA, Dufrêne YF. Staphylococcus aureus clumping factor a is a force-sensitive molecular switch that activates bacterial adhesion. Proc Natl Acad Sci. 2018;115(21): $5564-9$.

114. GO CM. db: a set of annotation maps describing the entire gene ontology; 2017.

115. Galili T. Dendextend: an R package for visualizing, adjusting and comparing trees of hierarchical clustering. Bioinformatics. 2015;31(22):3718-20.

116. Galili T, O'Callaghan A, Sidi J, Sievert C. Heatmaply: an R package for creating interactive cluster heatmaps for online publishing. Bioinformatics. 2018;34(9):1600-2.

117. R Core Team. R: A language and environment for statistical computing [internet]. Vienna: R Core Team; 2017. Available from: https://www.r-project. org/

118. Van Der ML. Accelerating t-SNE using tree-based algorithms. J Mach Learn Res. 2014;15:3221-45 Available from: http://jmlr.org/papers/v15/ vandermaaten 14a.htm|\%0A, http://jmlr.org/papers/v15/vandermaaten14a. html\%257B\%2525\%257D5Cnfiles/1017/JMLR-van\%257B_\%257Dder\%25 7B_\%257DMaaten-2014-Accelerating\%257B_\%257Dt-SNE\%257B_\%25 7Dusing\%257B_\%257DTree-Based\%257B_\%257DAlgorith.

119. Wickham H. ggplot2: elegant graphics for data analysis [internet]. New York: Springer-Verlag; 2016. Available from: https://ggplot2.tidyverse.org

\section{Publisher's Note}

Springer Nature remains neutral with regard to jurisdictional claims in published maps and institutional affiliations.

Ready to submit your research? Choose BMC and benefit from:

- fast, convenient online submission

- thorough peer review by experienced researchers in your field

- rapid publication on acceptance

- support for research data, including large and complex data types

- gold Open Access which fosters wider collaboration and increased citations

- maximum visibility for your research: over $100 \mathrm{M}$ website views per year

At $\mathrm{BMC}$, research is always in progress.

Learn more biomedcentral.com/submissions 